Revue des patrimoines

25 | 2014

Le patrimoine de la Grande Guerre

\title{
Muséographie(s) comparée(s) de la Grande Guerre en 2013. Allemagne - Belgique - France - Royaume- Uni
}

\section{Célia Fleury}

\section{(2) OpenEdition}

\section{Journals}

Electronic version

URL: http://journals.openedition.org/insitu/11559

DOI: 10.4000/insitu. 11559

ISSN: 1630-7305

\section{Publisher}

Ministère de la Culture

\section{Electronic reference}

Célia Fleury, «Muséographie(s) comparée(s) de la Grande Guerre en 2013. Allemagne - Belgique France - Royaume-Uni », In Situ [Online], 25 | 2014, Online since 29 December 2014, connection on 25 June 2020. URL : http://journals.openedition.org/insitu/11559; DOI : https://doi.org/10.4000/insitu 11559

This text was automatically generated on 25 June 2020.

\section{(c) $(7)$}

In Situ Revues des patrimoines est mis à disposition selon les termes de la licence Creative Commons Attribution - Pas d'Utilisation Commerciale - Pas de Modification 4.0 International. 


\title{
Muséographie(s) comparée(s) de la Grande Guerre en 2013. Allemagne - Belgique - France - Royaume-Uni
}

\author{
Célia Fleury
}

L'essentiel est de saisir à quel point la Grande Guerre transforma - et polarisa - les normes en vigueur dans différents domaines. [...] Ce qui explique également pourquoi, jusqu'à nos jours, il suffit de changer l'angle de vision pour retrouver

un aspect perdu ou découvrir une résonnance nouvelle à une guerre dont l'influence nous atteint encore ${ }^{1}$.

1 En lien avec des historiographies, souvent nationales, des conflits du $\mathrm{xx}^{\mathrm{e}}$ siècle, les parcours muséographiques qui traitent de la Grande Guerre au sein des musées d'histoire essayent de proposer des récits adaptés à leurs publics et à leur pré-requis éducatifs supposés. Mais le caractère international du conflit et l'approche du centenaire ont engendré un tourisme de mémoire démultiplié, notamment de la Flandre belge à la Somme, et de nouvelles formes d'appropriation des publics, notamment anglo-saxons, sensibles aux sites de mémoire ${ }^{2}$. La comparaison avec les muséographies de la Seconde Guerre mondiale ne peut donc être que partielle ${ }^{3}$ : il y a en effet probablement une spécificité des muséographies de la Grande Guerre, à la fois premier conflit mondial, et désormais sans témoin direct depuis 2011.

2 En revanche, y a-t-il des spécificités nationales en matière d'interprétation muséographique, comme il y en a en matière historiographique ? La comparaison d'un corpus d'une dizaine de musées des pays de l'Europe du nord-ouest impliqués dans la Première Guerre mondiale (Allemagne, Belgique, France et Royaume-Uni) permet de cerner davantage la provenance et les modalités de collecte des objets (armées, dépôts ou dons de particuliers, collections d'amateurs spécialisés, etc.), ainsi que leurs statuts au sein du musée (archives, documents, objets patrimoniaux, expôts ${ }^{4}$, etc.). Cet essai de 
typologie s'efforce donc de montrer comment ils traitent muséographiquement des aspects mémoriaux du conflit et s'ils développent des modalités d'interprétation en lien avec des sites de mémoire.

3 Y a-t-il confrontation entre plusieurs traditions muséographiques, transposées à l'interprétation de la Grande Guerre? L'une, davantage esthétisante et minimaliste, venue des Beaux-Arts, et l'autre d'immersion, visant plutôt l'expérience la plus complète possible du visiteur? Ces traditions muséographiques sont-elles nationales, ou plutôt liées à des effets de mode, nécessaires pour pérenniser la fréquentation des visiteurs? Afin de dépasser cette opposition entre muséographie d'objets et muséographie immersive $e^{5}$, comment une muséographie efficace peut être à la fois une muséographie d'objet (car l'objet est polysémique ${ }^{6}$ ) et une muséographie qui crée du lien entre les objets ? L'analyse de la muséographie est difficile, car à la fois résultat du processus lié à sa conception ${ }^{7}$, mais par essence, toujours en mouvement (prêt, déplacement d'objets, évolution des sections permanentes, etc.).

Figure 1

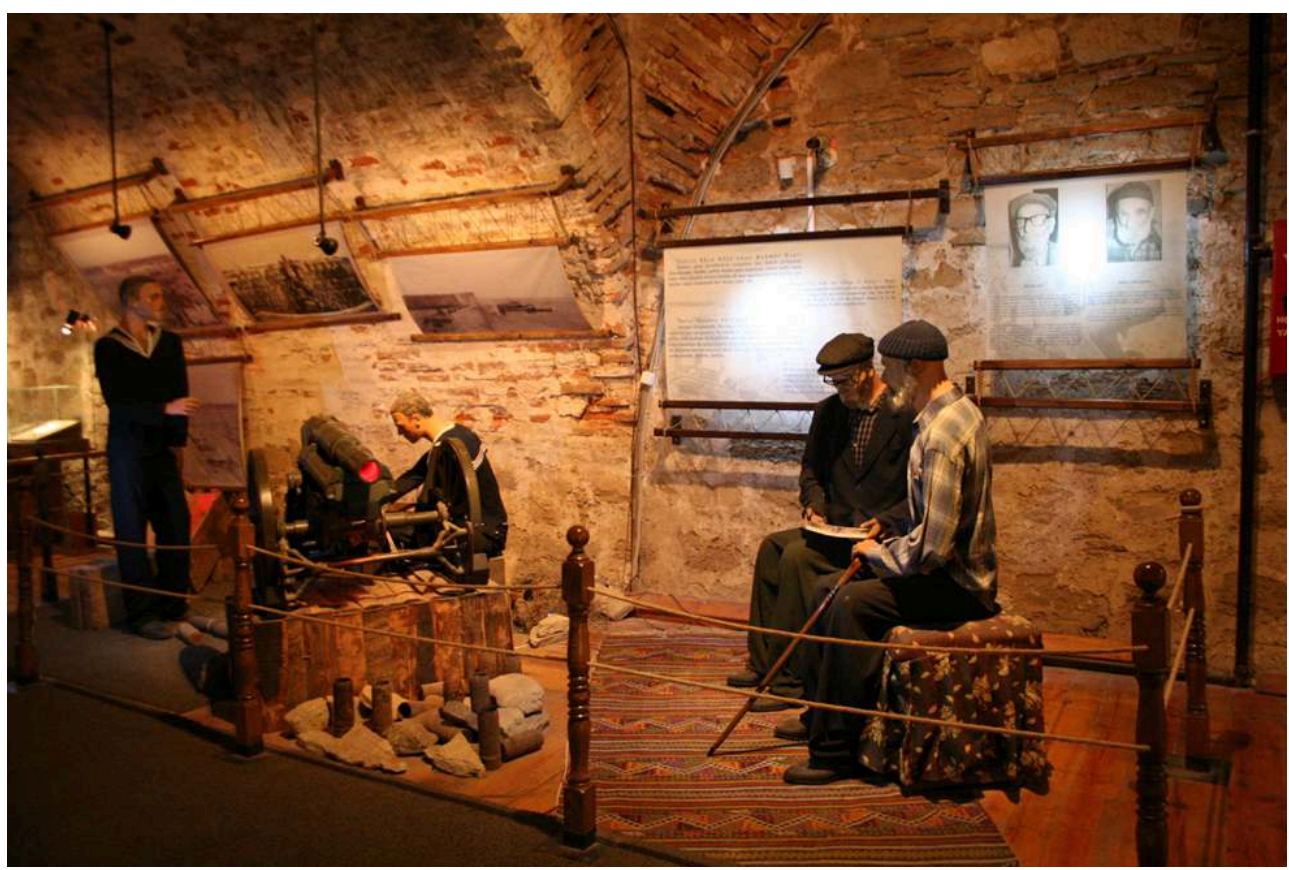

Saynète avec des mannequins représentant des anciens combattants qui ont témoigné de leurs expériences : leurs photos et biographies apparaissent sur le panneau explicatif accroché au mur. musée naval national turc, Çanakkale.

Phot. Fleury, Célia, février 2013. @ Célia Fleury.

Pour Massimo Baioni, il existe un langage spécifique aux musées d'histoire (fig. 1), "c'est-à-dire les dessins et les techniques de muséographie au moyen desquels fut pensée et construite la représentation de la guerre $»^{8}$.

L'exposition est plus que la somme de ses parties [, c'est] un enchevêtrement de sens, composé de multiples histoires qui pour finir permettent de produire une histoire, celle que le visiteur se construit par interaction avec les propositions [...] ce sont les visiteurs et eux seuls qui structurent la narration [...] c'est en fait un millefeuille, composé de couches de sens dans lequel le visiteur va voyager et glaner des niveaux qui lui conviennent. [...] L'exposition est donc un mode ouvert ${ }^{9}$. 


(1)

impossible, d'envisager une analyse exhaustive de l'ensemble des parcours muséographiques des huit musées concernés : Deutsches Historisches Museum (Berlin) et Militärhistorisches Museum der Bundeswehr (Dresde); Imperial War Museum (Londres); musée royal de l'Armée (Bruxelles) et In Flanders Fields Museum (Ypres) ; musée de la Grande Guerre du Pays de Meaux (Meaux), musée de l'Armée - Hôtel national des Invalides (Paris) et Historial de la Grande Guerre (Péronne). Parce que Thomas Thiemeyer précise que l'histoire de la muséographie de la guerre a souvent été faite sans les musées ${ }^{11}$, que le concept d' «entrepreneur muséal » est difficilement cernable à la lumière des enjeux opérationnels qui s'imposent à beaucoup de concepteurs de parcours muséographiques ${ }^{12}$, il semble important de comparer comment la Grande Guerre est traitée dans ces musées : les thèmes, les objets et les manières de les scénariser sont-ils similaires? En fait, il est nécessaire de travailler directement à partir du matériel muséographique (démarche inductive et analyse empirique), fondé sur la comparaison du traitement de certains thèmes récurrents (ou clés), dans le corpus des musées concernés. En effet, en utilisant la comparaison et la confrontation comme moyen de faire émerger la connaissance, se dessine un catalogue, même s'il n'est pas aussi raisonné que souhaité, des différents partis pris muséographiques d'interprétation à la veille du centenaire de la Grande Guerre.

7 Si pour analyser l'historiographie de la Grande Guerre, Antoine Prost et Jay Winter, qui ont pourtant fait l'effort de coécrire dans leurs propres langues maternelles, avouent les limites de leur analyse imposées par leurs connaissances linguistiques ${ }^{13}$, une immersion pendant trois mois dans une culture muséographique allemande ${ }^{14}$ a été nécessaire pour explorer une abondante bibliographie germanophone sur la muséographie de la Grande Guerre, peu exploitée jusqu'à présent par les muséologues anglophones ou francophones.

\section{Des traditions muséographiques nationales?}

8 Étrangement, peu de chercheurs, historiens ou muséologues francophones ${ }^{15}$, s'intéressent aux liens, voire aux influences entre les traditions historiographiques et muséographiques, comme si l'ouverture de l'Historial de la Grande Guerre à Péronne en 1992 avait résolu tous les questionnements théoriques et opérationnels possibles.

\section{Les historiographies de la Grande Guerre et les traditions muséographiques nationales}

«Enfin, comme il faut, par définition, être plusieurs pour faire la guerre, il aurait été absurde de n'examiner qu'une seule historiographie [...]» : analyser les composantes de l'historiographie internationale de la Grande Guerre est donc l'ambition d'Antoine Prost et de Jay Winter ${ }^{16}$. Sans être en capacité d'intégrer la bibliographie russophone ${ }^{17}$, dans leur premier chapitre, ils décrivent trois "configurations historiographiques » depuis la fin du conflit. Commune à la France, le Royaume-Uni et l'Allemagne, la première configuration " militaire et diplomatique » est une histoire vue « d'en haut », 
recherchant avant tout les « responsabilités de la guerre ${ }^{18}$, qui s'écrit sans les témoins directs, les poilus. La deuxième, "sociale ", à partir des années 1960, est notamment liée au «paradigme marxisme » et au rapport guerre/révolution, ainsi qu'à la diffusion de l'histoire de la Grande Guerre à la télévision qui a considérablement influencé l'historiographie anglo-saxonne et son thème privilégié du «monumental gâchis de la bonne volonté et du dévouement de millions d'hommes et de femmes». Enfin, la troisième a permis à l'histoire « culturelle » de la Grande Guerre d'émerger à partir de la fin des années 1980, tout en développant la «microstoria ou l'Alltagsgeschichte [...] qui ne sont pas les parties d'un tout mais méritent attention pour eux-mêmes ", dont l'influence est réelle dans les muséographies anglo-saxonnes et allemandes (fig. 2). La conclusion d'Antoine Prost et de Jay Winter montre clairement que «chaque culture nationale a développé des modèles narratifs » propres, en lien avec trois traditions différentes : « ironie britannique, cartésianisme français et sociologie weberienne ». En fait, l'historiographie anglo-saxonne insiste sur l'absurdité et la « futility » de la guerre, l'historiographie française s'est construite sur la nécessaire «survie de la collectivité nationale " liée à une "victoire douloureuse » et enfin, à cause d'une " défaite niée ", «l'historiographie allemande se structure plus tardivement » à cause de la Deuxième Guerre mondiale ${ }^{19}$.

Figure 2

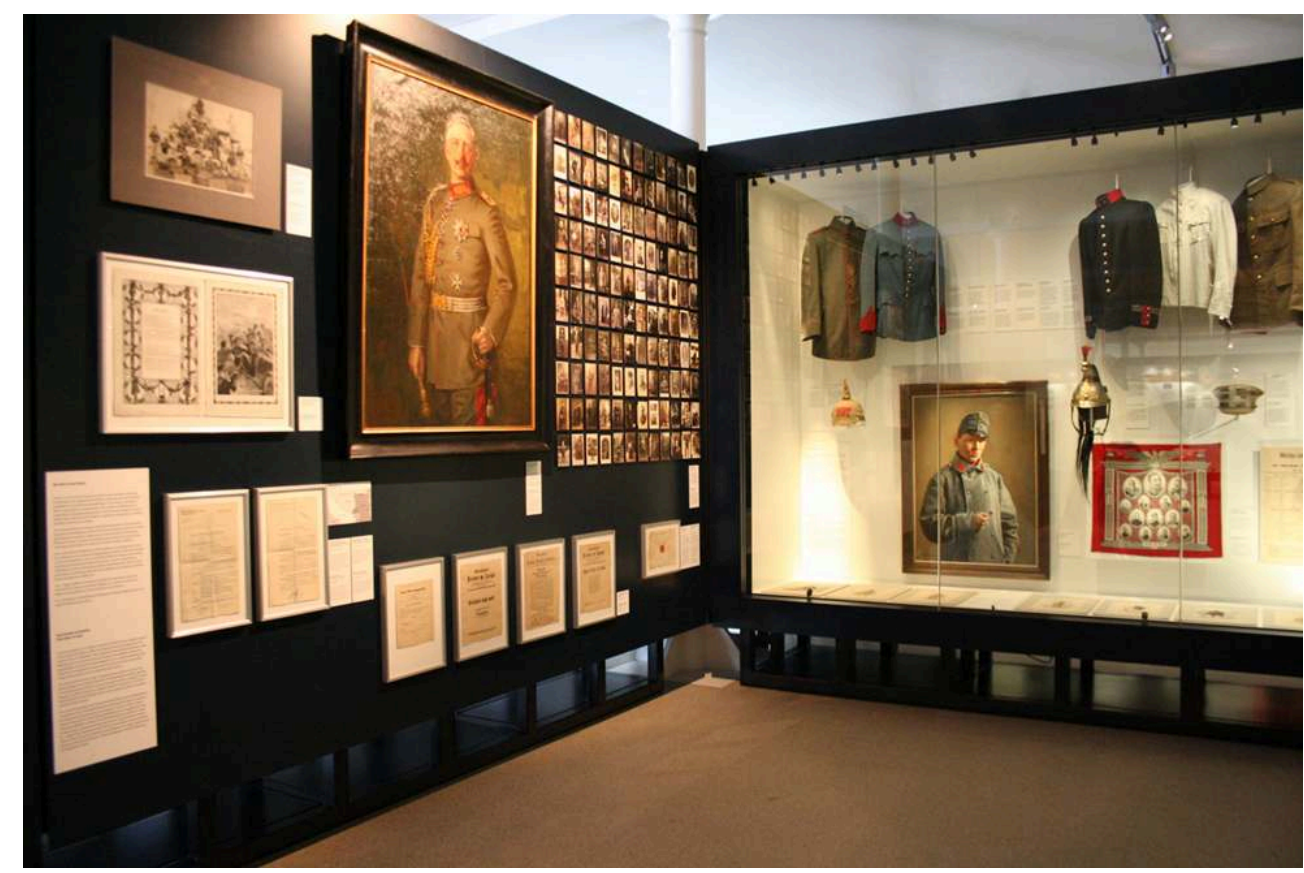

Vue du premier espace de la section Grande Guerre, dont mur de photos individuelles originales de soldats. Militärhistorisches Museum der Bundeswehr, Dresde.

Phot. Fleury, Célia, juin 2013. (c) Célia Fleury.

10 Ces grandes tendances historiographiques, parfois historiquement datées ou imbriquées, participent aussi à la construction de cultures mémorielles nationales, voire à des imaginaires collectifs différenciés, et inversement. L'ouvrage de Pierre Nora est bien connu des chercheurs anglophones et germanophones ${ }^{20}$; en parallèle des Memory studies, l'analyse de la Erinnerungskultur est particulièrement développée par les historiens allemands qui analysent les muséographies de la Grande Guerre ${ }^{21}$. Ces 
cultures mémorielles, dont probablement une partie de l'histoire est encore à écrire ${ }^{22}$, même si souvent les sources manquent ${ }^{23}$, ont fatalement influencé la création de musées et de leurs parcours muséographiques dans les pays concernés ${ }^{24}$. Le " délicat enchevêtrement qui préside au rapport entre le deuil public et le deuil privé $»^{25}$ expliquerait probablement les différences de cultures mémorielles entre les AngloSaxons, les Français et les Allemands. En effet, les soldats anglo-saxons ont été enterrés sur place et l'Imperial War Museum de Londres a été créé dès $1920^{26}$. Pour les Allemands, le concept historiographique de Urkatastrophe (catastrophe originelle) ${ }^{27}$ traverse les commentaires de beaucoup de panneaux de salle. Enfin, les Belges, malgré la neutralité puis l'occupation de la Belgique pendant la guerre, essayent d'expliquer aux visiteurs leurs spécificités. Par ailleurs, certains pays sont devenus politiquement autonomes, suite à la Grande Guerre, qui occupe ainsi dans leurs histoires respectives une place essentielle, comme d'ailleurs dans leurs cultures mémorielles nationales : les membres du Commonwealth, le Canada, la Nouvelle-Zélande et l'Australie (ANZAC), mais aussi la Turquie (fig. 3). Renouvelée depuis les années 1980 et très vive, la culture mémorielle australienne, par exemple, a inspiré récemment le gouvernement australien dans la construction d'un chemin de mémoire australien sur le front ouest, ponctué d'espaces muséographiques, de même qu'à Gallipoli²8.

Figure 3

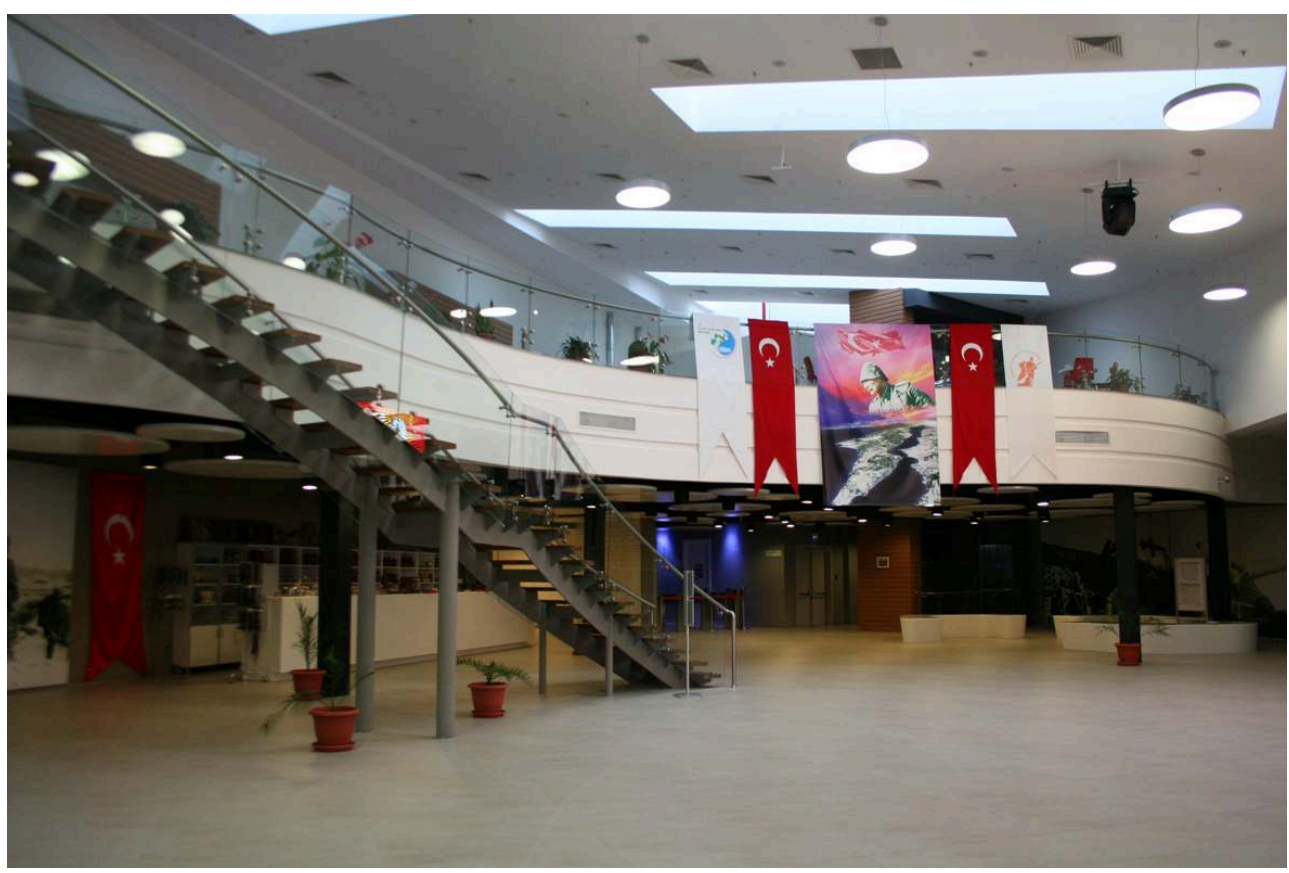

Hall d'accueil du centre d'interprétation Canakkale Destani ouvert en juin 2012. Canakkale Destani, Péninsule de Gallipoli.

Phot. Fleury, Célia, février 2013. @ C Célia Fleury.

11 Ces traditions historiographiques et mémorielles différenciées, notamment la microstoria, la distance entre les lieux d'inhumation et les familles, ainsi que la précocité de l'Imperial War Museum de Londres, expliqueraient probablement que la muséographie immersive d'expérience de la guerre, soit d'origine anglo-saxonne. Les nombreux commentaires négatifs francophones sur la fameuse Trench Experience (tranchée du Tommy en 1916) ${ }^{29}$, qui n'est plus accessible au public depuis juillet 2013, 
montrent effectivement que les cultures et les tabous muséographiques sont aussi nationaux. Pourtant, proposer une immersion totale dans la guerre, expérience physique (aussi sonore et olfactive) a priori non vécue réellement par les visiteurs occidentaux du xxi ${ }^{e}$ siècle, est un enjeu pédagogique important pour les Anglo-Saxons comme le montre l'Étude sur la transmission de la mémoire de la Grande Guerre par le biais d'outils pédagogiques ${ }^{30}$ : pour les musées anglo-saxons, c'est en effet un moyen de créer du lien entre les générations et d'héroïser les individus ${ }^{31}$. Par exemple, il existe une reconstitution de tranchée du front occidental dans la section dédiée aux enfants de l'Australian War Memorial (Discovery zone) où ils peuvent manipuler des objets authentiques (fig. 4) et prendre connaissance d'histoires individuelles réelles (voir fig. 1); un atelier dédié aux scolaires au musée canadien de la guerre d'Ottawa est similaire. De même, pour répondre aux attentes des scolaires britanniques qui viennent sur le site de la bataille d'Ypres, le Memorial Museum Passchendaele 1917 leur propose "The Platoon experience » qui, par le moyen d'un parcours de trois kilomètres, tente de leur montrer que «la guerre n'est pas un jeu ». Est-ce que les débats français sur l'impossible mélange entre mémoire et histoire, ou entre muséographie d'immersion et d'objets sont si pertinents ${ }^{32}$ aux yeux des Anglo-Saxons ? Côté allemand, même si aucun musée n'est consacré exclusivement à la Grande Guerre, une importante bibliographie sur la muséographie des musées d'histoire ${ }^{33}$, et tout particulièrement des guerres, existe depuis les années 1990. Si un important colloque sur les musées de guerre, publié en $1997^{34}$, semble être le début d'une importante réflexion universitaire, deux chercheurs allemands, Christine Beil ${ }^{35}$, et Thomas Thiemeyer ${ }^{36}$, sont incontournables sur l'histoire de la muséographie des musées de guerre ${ }^{37}$, la scénographie de la violence, et les liens entre authenticité, esthétisation, objectivité et fascination pour la technique et les armes au musée. 
Figure 4

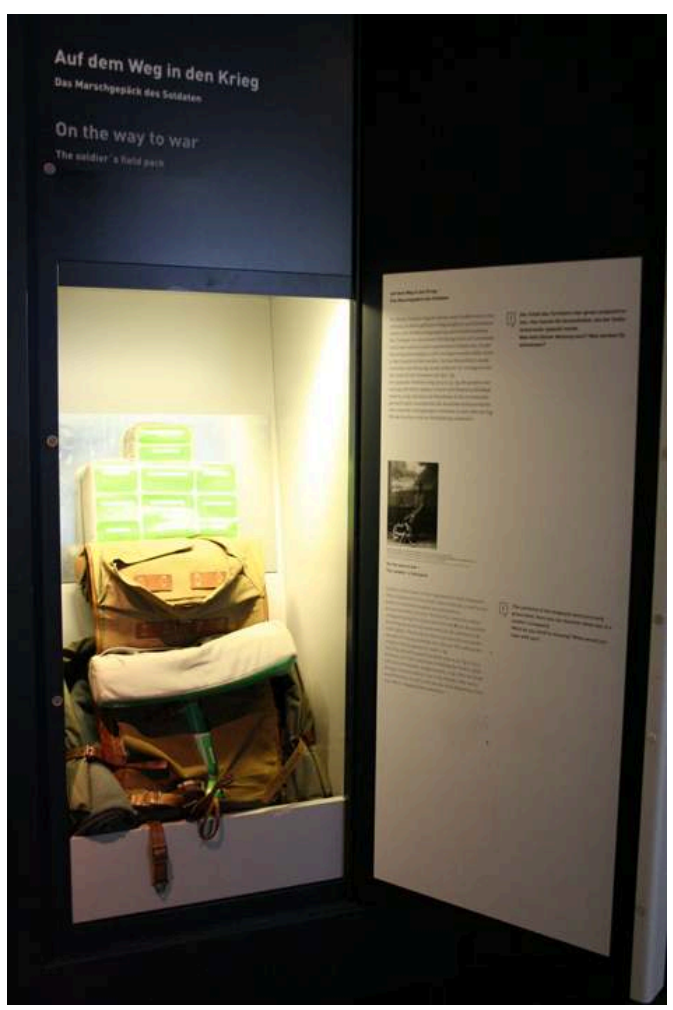

Un sac à dos de soldat que le visiteur peut essayer et soupeser dans la section Grande Guerre. Militärhistorisches Museum der Bundeswehr, Dresde.

Phot. Fleury, Célia, juin 2013. (c) Célia Fleury.

\section{Des musées militaires nationaux et des musées d'histoire territoriaux}

12 L'historiographie de la Grande Guerre et l'histoire de ses cultures mémorielles expliqueraient une construction muséographique en deux temps, d'abord un traitement dans les musées militaires nationaux, puis beaucoup plus tardivement, dans des musées d'histoire territoriaux. Cette différence fondamentale paraît être sousestimée par Thomas Thiemeyer ${ }^{38}$.

L'objet n'est pas de revenir sur l'histoire des musées militaires nationaux (Militärhistorisches Museum der Bundeswehr de Dresde ${ }^{39}$, Imperial War Museum de Londres ${ }^{40}$, musée royal de l'Armée de Bruxelles ${ }^{41}$ et musée de l'Armée - Hôtel national des Invalides de Paris ${ }^{42}$ ), largement traitée par ailleurs, mais de la prendre en compte lors de la comparaison des muséographies contemporaines. En effet, leurs histoires et l'histoire de leurs collections conditionnent en partie le discours que ces musées peuvent proposer aux publics aujourd'hui. Si l'Imperial War Museum (Londres) est la création la plus ancienne, en 1920, probablement parce que l'armée anglaise avait peu développé son infanterie (armée de terre) avant la Grande Guerre (fig. 5), l'ensemble des autres musées nationaux des armées ont des origines royales ou princières antérieures $^{43}$. Retracée très clairement par Isabelle Benoît ${ }^{44}$, l'histoire des arsenaux militaires allemands (Prusse, Bavière, Saxe, Bade) depuis le XVIII siècle est assez explicite : à l'ouest, les collections d'armes sont progressivement regroupées à Rastatt, 
à partir des années 1980, en vue de créer un musée de la Bundeswehr. Une partie de leurs collections provient de ce qui pourrait être assimilé au « dépôt légal » des armées, permettant aux militaires d'avoir des objets historiques de référence sur leur histoire ${ }^{45}$. D'ailleurs, il existe une similitude certaine dans les bâtiments de ces musées, souvent classiques et néoclassiques, adossés de matériels militaires imposants que le promeneur, potentiel visiteur, ne peut manquer ; l'architecte Daniel Libeskind joue la rupture contemporaine avec ceux sur lesquels il intervient ${ }^{46}$. Quelques auteurs s'accordent pour écrire que rares sont les musées militaires nationaux qui s'efforcent exclusivement de produire un discours historique - mais ce n'est pas forcément leur première vocation -, même si la section sur la Grande Guerre du musée de l'Armée de Paris semble faire un effort significatif dans ce sens, en essayant d'intégrer largement les grandes tendances de l'historiographie, dont culturelle ${ }^{47}$.

Figure 5

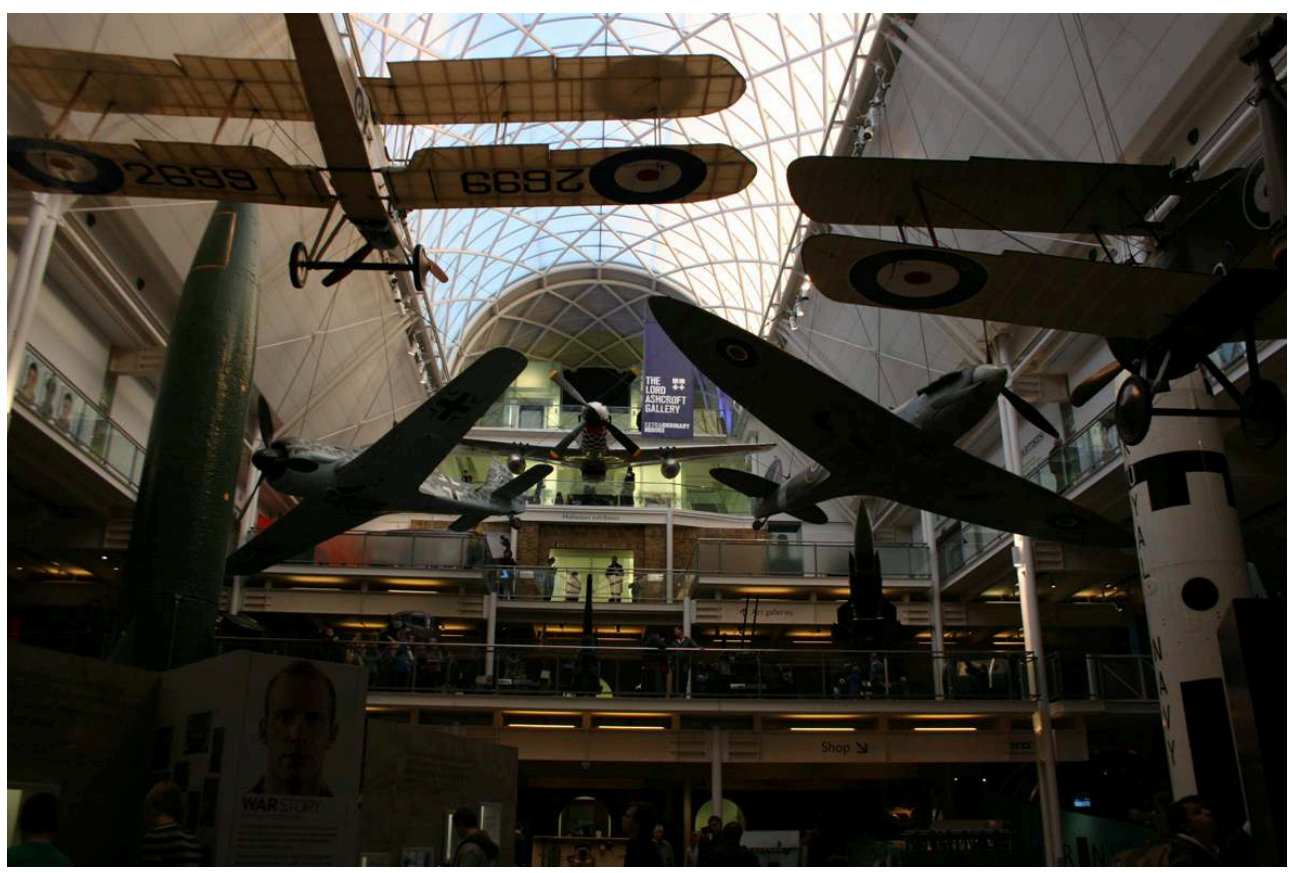

Hall d'entrée avec des avions réels. Imperial War Museum, Londres.

Phot. Fleury, Célia, novembre 2011. (c) Célia Fleury.

En revanche, les musées de la Grande Guerre territoriaux, situés sur l'ancien front ouest (In Flanders Fields Museum d'Ypres, musée de la Grande Guerre du Pays de Meaux et Historial de la Grande Guerre de Péronne) sont beaucoup plus récents, et correspondent à la dernière vague de création, liée notamment à l'implication des collectivités territoriales ${ }^{48}$. Contrairement aux musées nationaux des armées, leurs collections n'ont pas été originellement constituées par du matériel militaire. Cependant, les choix et les politiques d'acquisition de ces musées ne sont pas pour autant similaires : la collecte auprès de la population a toujours été au cœur de la politique d'acquisition d'Ypres ${ }^{49}$, tandis que Péronne a « illustré » son premier parcours muséographique à partir d'objets trouvés sur le marché50, et Meaux a été créé à partir de la collection de Jean-Pierre Vernet ${ }^{51}$. En fonction de leur ancrage, ces musées de la Grande Guerre territoriaux essayent de développer une ouverture muséographique vers les lieux de batailles et de mémoire proches : à Ypres, vers les lieux des différents 
batailles et les cimetières correspondants (par exemple, Tyne Cote, le plus important cimetière de la Commonwealth War Graves Commission en Europe, ou celui allemand de Vlaslo); à Meaux, des gros hublots au sein de l'espace muséographique permettent de voir le sol extérieur cartographié (fig. 6) et le toit-terrasse permet de voir le monument américain, La Liberté éplorée, inauguré en 1932. Sans se présenter comme "musées-mémoriaux», certains dépassent l'interprétation historique de la Grande Guerre en présentant des espaces scénographiés quasi mémoriels : par exemple, le centre d'interprétation du Mémorial de Tiepval dépend directement de l'Historial de la Grande Guerre de Péronne, et à Meaux, le dernier couloir vers la sortie propose une ambiance sombre quasi mémorielle ${ }^{52}$.

Figure 6

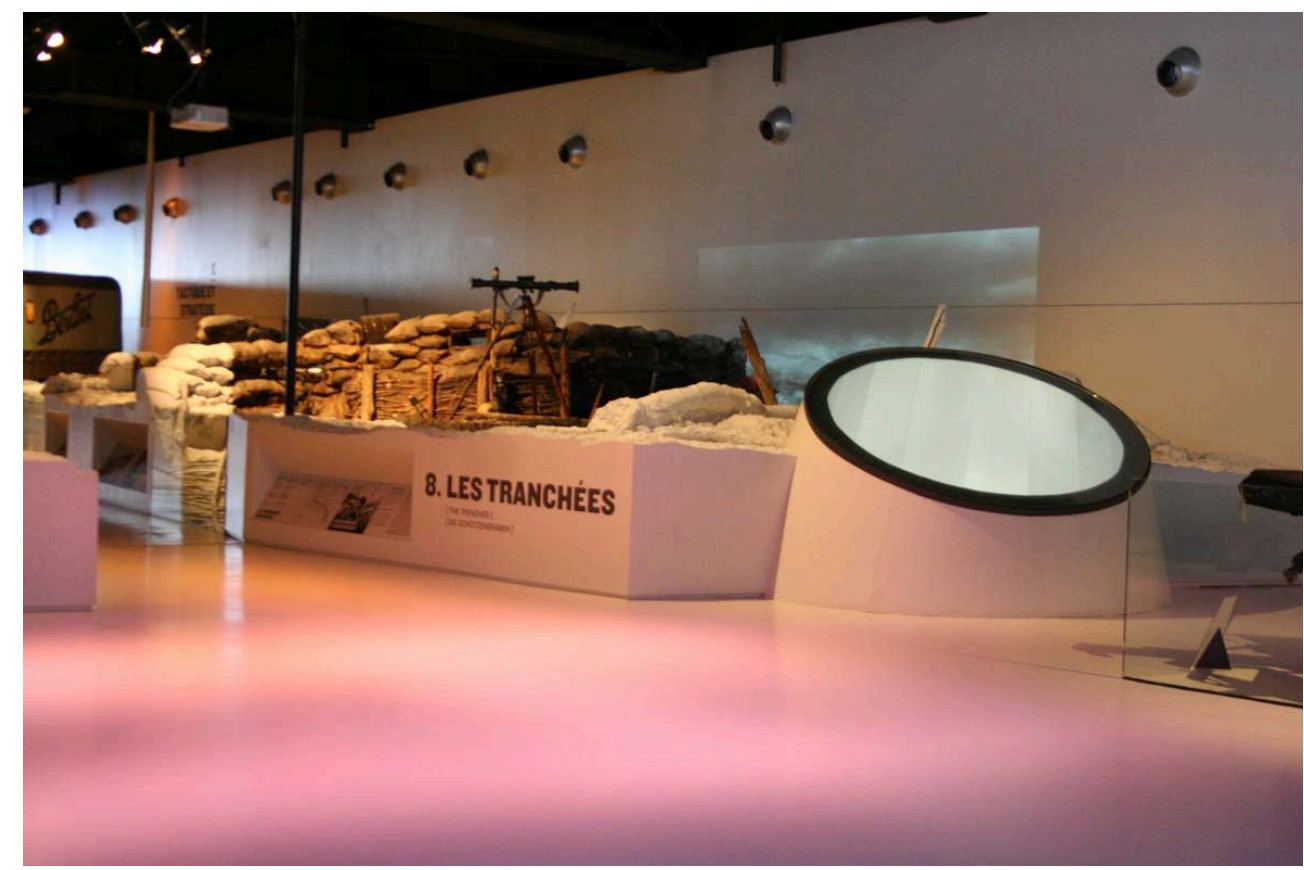

Entrée de la section sur la guerre de tranchée avec un hublot qui permet une vue sur le sol extérieur cartographié. musée de la Grande Guerre du Pays de Meaux, Meaux.

Phot. Fleury, Célia, août 2013. (c) Célia Fleury.

Peut-être font exception à cette classification, étroitement liée à l'histoire des musées de la Grande Guerre, deux musées : le Deutsches Historisches Museum (Berlin) ${ }^{53}$ et le musée naval national turc (Çanakkale, en face de la péninsule de Gallipoli). Contrepoint au corpus initial, ce dernier présente la spécificité d'être le seul musée naval, et sur site, au niveau du détroit des Dardanelles, lieu d'importants combats en 1916 et 1917 entre la France, le Royaume-Uni et l'Empire ottoman. Présenté comme le seul musée historique par Thomas Thiemeyer ${ }^{54}$, le Deutsches Historisches Museum (Berlin) a une histoire mouvementée : dans le bâtiment Zeughaus, ancien arsenal prussien sur Unter den Linden, ses concepteurs n'ont pas voulu en faire un musée militaire national, mais bien un musée d'histoire nationale. Projet imaginé par la RDA, il a fait l'objet de nombreuses controverses après la chute du mur de Berlin $^{55}$ : ses objectifs et ses collections sont donc intimement liés à la réunification de l'Allemagne. Le fonds ancien est quasiment inexistant, même si quelques restitutions ont pu être négociées avec la France et la Russie ${ }^{56}$ : les collections ont donc été constituées récemment en vue de 
répondre aux besoins du discours historique, un peu comme pour l'Historial de la Grande Guerre (Péronne) ${ }^{57}$. Si l'Allemagne propose à ses concitoyens et aux touristes une muséographie de son histoire complexe depuis l'Antiquité (fig. 7), le musée naval national turc est situé sur un haut lieu de l'histoire navale de l'Empire ottoman : il est donc volontairement territorialisé, le site explicitant ainsi l'émergence d'une nouvelle nation, l'État turc se défendant légitimement contre une invasion impérialiste. musée naval, il présente en extérieur plusieurs éléments de sa collection, mais surtout la reconstitution du bateau qui a permis la pose de mines sous-marines, empêchant les navires militaires français et britanniques d'entrer dans les Dardanelles en $1916^{58}$. Ce musée naval national turc, dont le parcours muséographique se développe majoritairement sur les trois niveaux du château du $\mathrm{xv}^{\mathrm{e}}$ siècle, est complémentaire au centre d'interprétation (avec un espace muséal) situé sur la péninsule de Gallipoli, ouvert depuis juin 2012 (voir fig. 3) ${ }^{59}$, mais leurs discours interprétatifs valorisent le courage des soldats, turcs et ANZAC, et l'émergence de l'État turc, grâce à la figure de Mustafa Kemal, qui faisait alors partie de l'état-major ottoman. Ces deux exemples montrent comment la Grande Guerre est partie prenante, dans la muséographie de l'histoire nationale, et non pas seulement militaire.

Figure 7

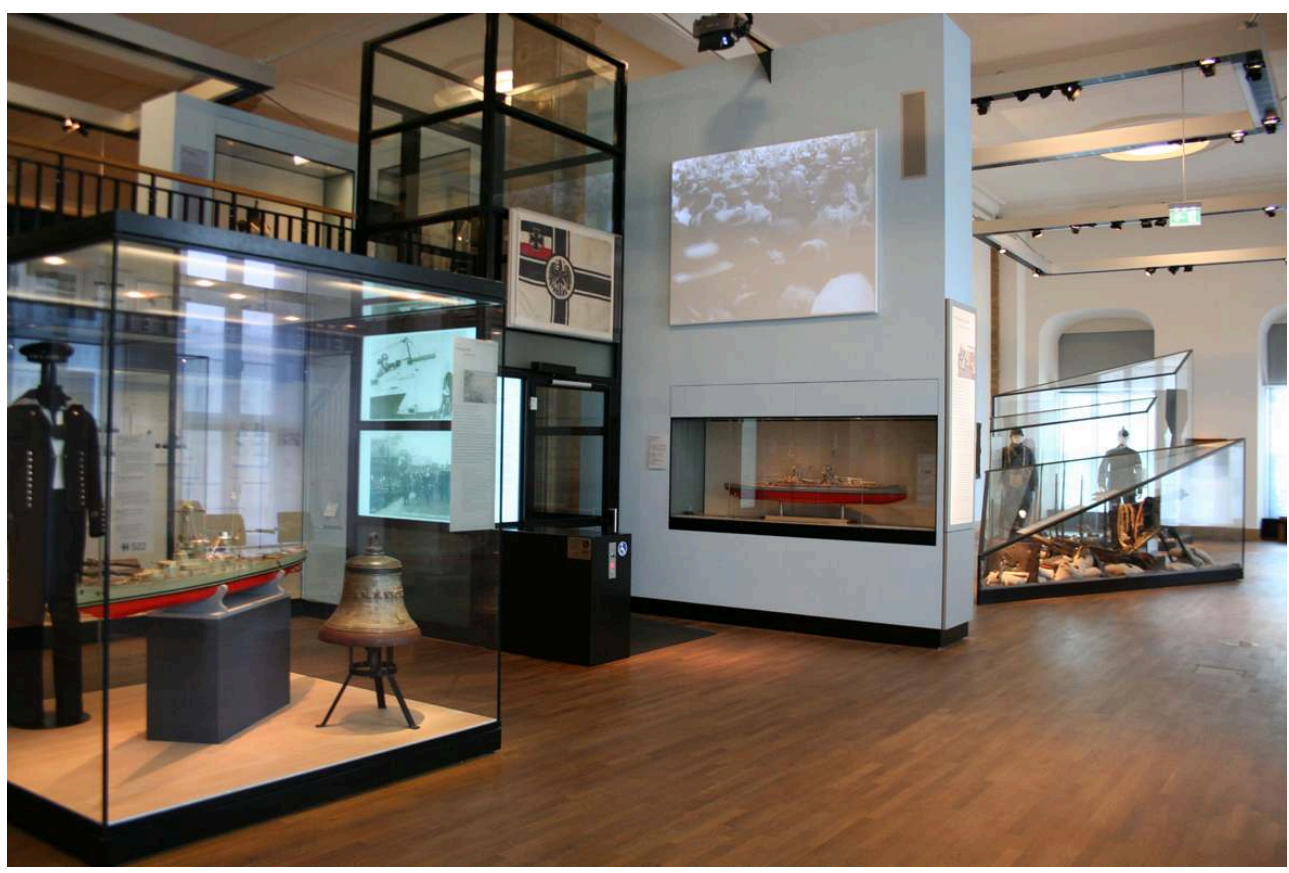

Transition entre la galerie du xix ${ }^{\mathrm{e}}$ siècle et la section Grande Guerre. Au premier plan, maquette de navire et sa cloche authentique. Deutsches Historisches Museum, Berlin.

Phot. Fleury, Célia, mai 2013. (c) Célia Fleury.

\section{Le succès muséographique et bibliographique de l'Historial de la Grande Guerre de Péronne}

Antoine Prost et Jay Winter avouent d'emblée que leur ouvrage commun n'est qu'une « introduction à ce que pourrait être l'histoire européenne de la Première Guerre mondiale, qu'il faudra bien écrire un jour si l'Europe se forge une identité $»^{60}$. 
Si l'histoire européenne de la Grande Guerre n'est pas encore écrite, l'Historial de la Grande Guerre (Péronne) est-il parvenu à combler un double manque, historiographique et muséographique? Fermé le 31 décembre 2013 pour une réouverture renouvelée le $1^{\mathrm{er}}$ mars 2014, la muséographie de l'Historial de Péronne a exceptionnellement tenu vingt ans (fig. 8) : cette longévité est gage d'un sérieux et d'un concept historique et muséographique mûri' ${ }^{61}$. Pour Massimo Baioni, l'Historial est le « résultat le plus ambitieux et le plus réussi de la volonté de privilégier une approche traduisant sur le plan muséographique la dimension sociale et totale du conflit, en accord avec les thématiques explorées par l'historiographie la plus récente ${ }^{62}$. La constitution d'un comité scientifique transnational lui a évité de tomber dans les travers d'une historiographie de la Grande Guerre ${ }^{63}$, encore trop nationale, mais pour autant, son discours historique était-il européen ou universaliste? C'est par la comparaison des objets nationaux que la muséographie parvenait à garder une distanciation nécessaire ${ }^{64}$. Rejetant une muséographie trop immersive, de tradition anglo-saxonne (Londres, Çanakkale), le succès bibliographique de l'Historial s'explique probablement par l'intégration des réflexions allemandes en matière d'interprétation historique, ce qui expliquerait que sa muséographie ait influencé beaucoup d'expositions allemandes des années 1990, comme le montre Suzanne Brandt ${ }^{65}$. De quelles manières la muséographie de l'Historial en a-t-elle influencé d'autres, parfois de manière paradoxale?

Figure 8

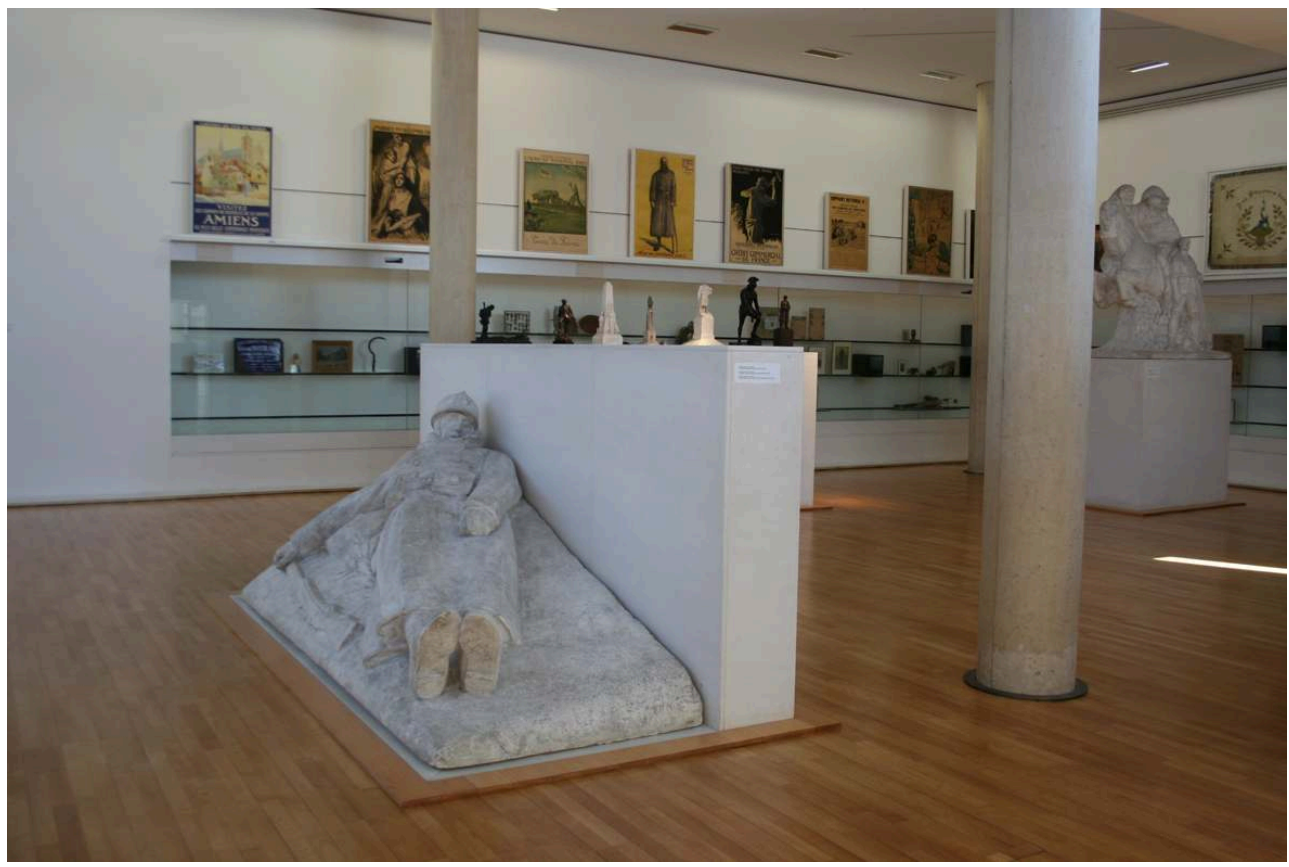

Salle de l'après-guerre (muséographie avant décembre 2013). Historial de la Grande Guerre, Péronne. Phot. Fleury, Célia, juin 2011. @ Célia Fleury.

Avec une constitution de collection de tradition plus anglo-saxonne, grâce à de multiples et médiatiques collectes, In Flanders Fields Museum (Ypres) a choisi de rendre sa nouvelle muséographie, accessible au public depuis juin 2012, moins immersive et plus riche de références et de renvois aux territoires environnants. Même si le visiteur peut toujours, s'il le souhaite, s'identifier à un témoin réel de la Grande 
Guerre $^{66}$, comme le proposait aussi la muséographie antérieure de 1998 à $2011^{67}$, il parcourt un espace muséographié moins foisonnant: par exemple, seule une musique contemporaine, composée exclusivement pour le lieu, constitue la nouvelle ambiance sonore (avant 2011, les sources sonores étaient beaucoup plus variées : extraits de documents sonores originaux, lectures de textes, dont poèmes, bruits de bombardements, etc. ${ }^{68}$. En revanche, grâce à un savoir-faire muséographique hors pair (les images et les sons provenant des différentes sections ne s'annulent pas, mais permettent la création d'ambiances homogènes et originales), cette seconde muséographie d'Ypres développe plusieurs niveaux de lecture grâce à de multiples ressources, parfaitement hiérarchisées (documents personnels, photos aériennes, témoignages joués par des acteurs - (voir fig. $\left.{ }^{\circ} 15\right)$-, projections, etc.). Dans cette muséographie, l'objet muséal peut reprendre complètement sa place de source (fig. 9) (voir fig. 14) ${ }^{69}$. Et pour gérer la masse d'objets si spécifiques à la période de la Grande Guerre, le conservateur de musée a parfois recours à d'autres compétences : l'expertise reste souvent encore chez des collectionneurs, qui ont accumulé pendant des décennies une connaissance liée à la manipulation et à la matérialité de ces objets ${ }^{70}$.

Figure 9

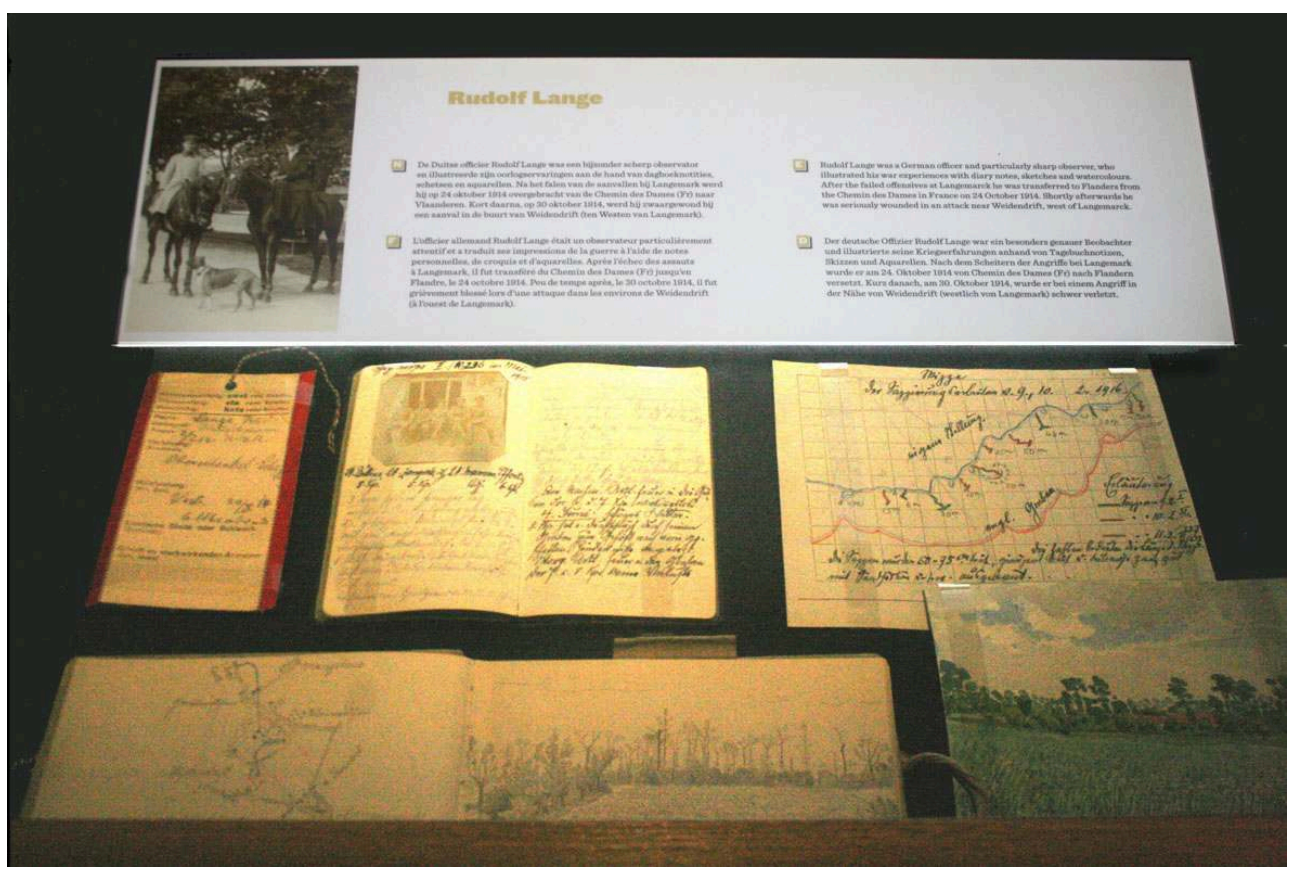

Une des vitrines dédiées aux objets de Rudolf Lange, officier allemand, avec notamment ses carnets de croquis. In Flanders Fields Museum, Ypres.

Phot. Fleury, Célia, décembre 2013. @ Célia Fleury.

D'une certaine manière, un peu paradoxalement, compte tenu de toutes les réserves des muséologues qui analysent les musées d'histoire, les objets semblent imposer leur matérialité dans la muséographie de la Grande Guerre. Même si contrairement à Péronne, la constitution de leurs collections a précédé la création d'un discours historique et de son scénario muséographique, les deux parcours les plus récents, le Militärhistorisches Museum der Bundeswehr (Dresde) et le musée de la Grande Guerre $\mathrm{du}$ Pays de Meaux (Meaux), ouverts respectivement au public en octobre 2011 et en novembre 2012, proposent une muséographie délibérément d'objets, parfois 
esthétisante (voir fig. 2), sans aucune immersion grâce à des espaces factices, comme à Londres, Ypres ou Çanakkale. La frontière même avec des installations d'art contemporain est parfois floue: si Dresde pose d'emblée l'art contemporain comme partis pris mémoriel d'interprétation dès l'entrée dans le parcours chronologique, l'intégration de mise en scène d'objets de guerre au sein du parcours muséographique (fig. 10) n'est pas sans questionner le visiteur averti - scénographie ou art contemporain ? -. Les galeries thématiques au sein de l'extension contemporaine étant délibérément plus esthétiques que de contenu historique ${ }^{71}$. La galerie initiatique du musée juif de Berlin, dans le bâtiment contemporain de Daniel Libeskind, et l'installation Shalechet de l'artiste israélien Menashe Kadishman la clôturant ${ }^{72}$ lui donnait un véritable caractère de "musée-mémorial ${ }^{73}$, ce qui n'est pas le cas de Dresde. En revanche, l'esthétisation d'objets ou d'assemblages, à l'entrée des deux parcours sur la Grande Guerre, paraît avoir une véritable valeur symbolique ou synecdotique : sur fonds rouges, le «cavalier de l'Apocalypse » des temps modernes et le morceau de pain du siège de 1871 remplacent bien des discours historiques. Quels seront les nouveaux partis pris de l'Imperial War Museum (Londres) à l'été 2014 ?

Figure 10

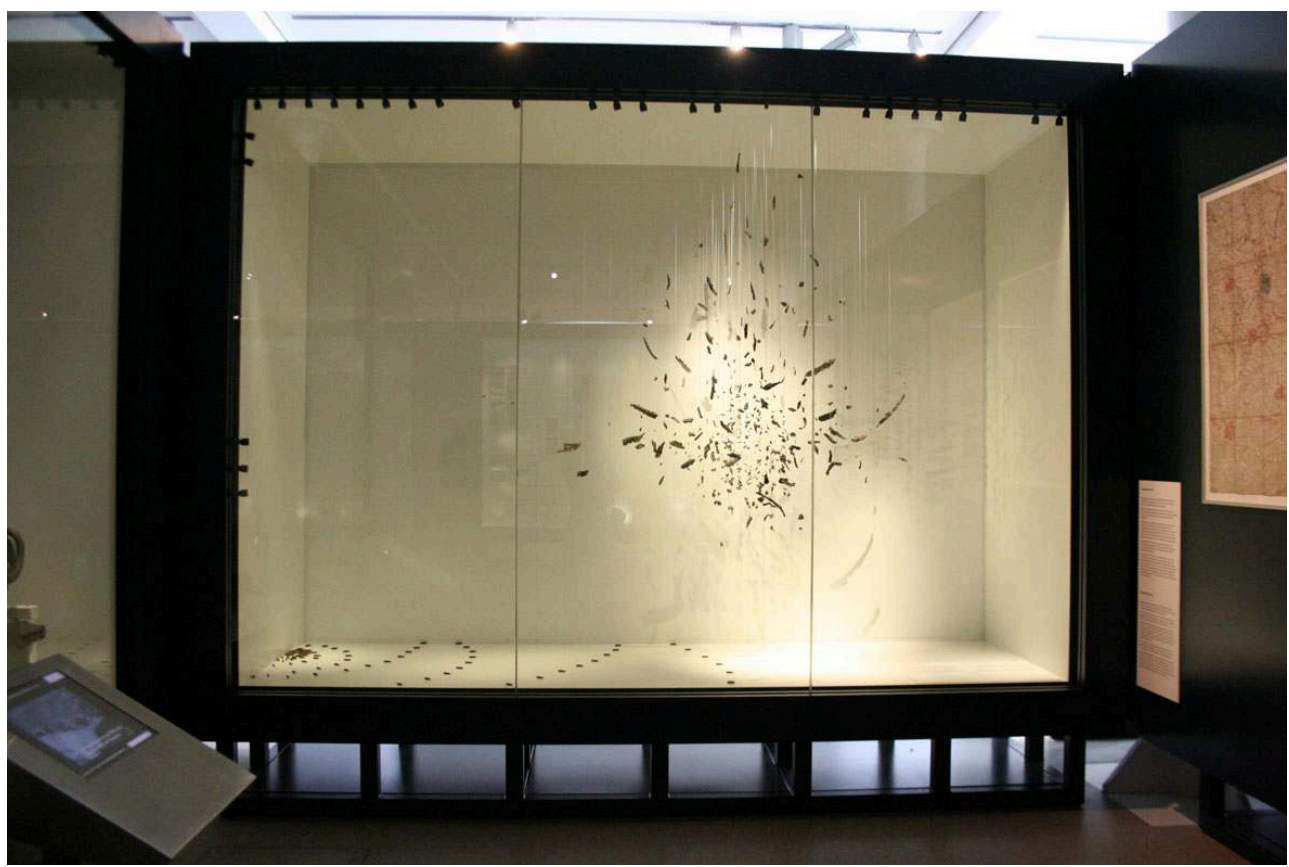

Vitrine de la galerie thématique des deux guerres mondiales intitulée « Geschossgarbe und Splitterhagel » (rafale et grêle d'éclats d'obus). Le cartel détaille les effets destructeurs des éclats d'obus. Militärhistorisches Museum der Bundeswehr, Dresde.

Phot. Fleury, Célia, juin 2013. (c) Célia Fleury.

\section{Quelles récurrences thématiques et scénographiques en 2013 ?}

Après avoir analysé les différents contextes de création de parcours muséographiques traitant de la Grande Guerre et leurs différentes traditions nationales, il convient d'esquisser une comparaison thématique des différents musées du corpus, révélant des 
choix muséographiques. Même si l'historiographie n'est pas encore européenne, des thèmes similaires, illustrés par des objets comparables, scandent ces parcours muséographiques.

\section{Les soldats et le front}

L'enjeu est de parvenir à représenter l'ampleur et la diversité de la mobilisation dans des espaces aux volumes restreints. Plus que le réalisme des mannequins, ce sont les uniformes, par l'évolution et la nationalité de leurs formes et de leurs couleurs, qui permettent aux différentes muséographiques d'illustrer cette thématique. Au musée de la Grande Guerre du Pays de Meaux et au musée de l'Armée de Paris, les mannequins vêtus de divers uniformes, souvent à l'échelle humaine, s'accumulent dans de gigantesques vitrines : à Meaux, le visiteur est même invité à la traverser, grâce un passage obligé entre les sections qui traitent de l'avant-guerre et la galerie principale ${ }^{74}$. En parallèle de cet effet de masse qui rend difficile une appréhension précise des différents corps de soldats, d'autres musées, comme le Deutsches Historisches Museum de Berlin, le musée royal de l'Armée de Bruxelles et le In Flanders Fields Museum d'Ypres, utilisent le porte-manteau comme synecdoque à l'uniforme complet, rendant possible la suspension de nombreux d'entre eux vides, les détachant ainsi symboliquement des hommes qui les ont portés (voir fig. 2). De même à Berlin, Dresde ou Ypres, les casques, vides et exposés seuls, remplissent aussi cette fonction. Deux musées, celui d'Ypres et l'Historial de la Grande Guerre de Péronne, parviennent à scénariser l'ensemble de l'équipement militaire des soldats des différentes nationalités : le premier, horizontalement, plutôt au début de l'évocation du conflit, évoquant des corps inertes allongés sur le sol ${ }^{75}$, et le second, verticalement, plutôt à la fin du parcours, donnant davantage de monumentalité à ce qui constituait l'attirail du soldat (fig. 11). Le Militärhistorisches Museum de Dresde propose même au visiteur, grâce à une armoire pédagogique intégrée au sein du parcours, de soupeser le sac à doc d'un soldat de la Grande Guerre (voir fig. 4). Les armes lourdes sont souvent traitées muséographiquement à part : l'effet d'accumulation scénarisée cherche à les présenter dans un contexte militaire offensif comme à Bruxelles et Péronne. Mais hors contexte, l'accumulation, par exemple des séries de fusils identiques dans une même vitrine en position horizontale de combat, comme à Dresde et Paris, contribue aussi à proposer une interprétation au second degré. 
Figure 11

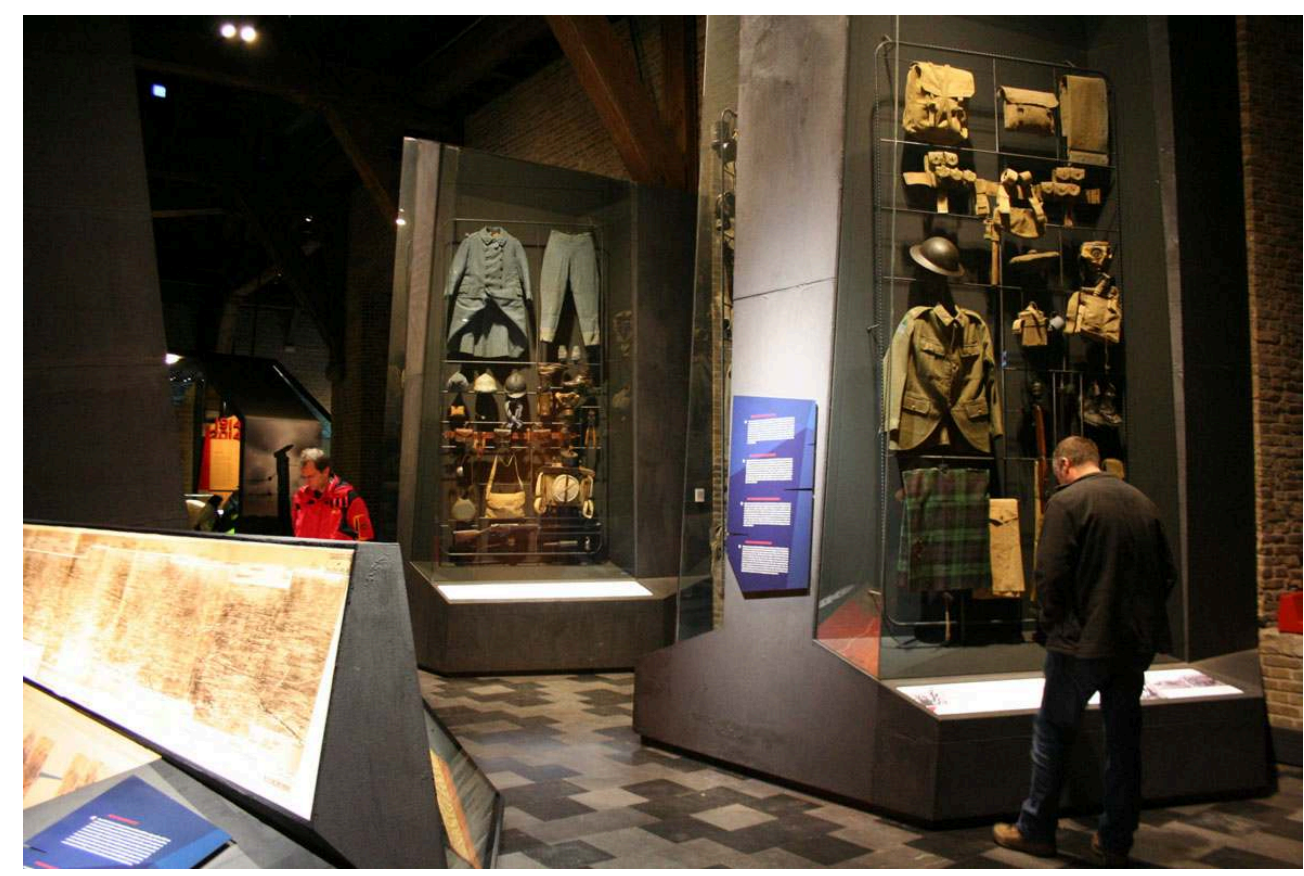

Équipements militaires de soldats de différentes nationalités. In Flanders Fields Museum, Ypres.

Phot. Fleury, Célia, décembre 2013. ㄷ Célia Fleury.

Si l'Imperial War Museum de Londres et le musée naval national turc de Çanakkale sont allés loin dans la reconstitution de tranchées immersives permettant aux visiteurs d'entendre les sons de la guerre ${ }^{76}$, ils ont en fait intégré dans leurs parcours muséographiques intérieurs ce que d'autres font visiter en extérieur, par exemple, les tranchées originales conservées et régulièrement entretenues au Mémorial canadien de Vimy (Pas-de-Calais). L'archéologie de la Grande Guerre ${ }^{77}$, évoquée à Ypres dans la section traitant des tranchées, devient aussi expérimentale, si bien que le Memorial Museum Passchendaele 1917 de Zonnebeke et le Fort Leveau à Feignies reconstituent en extérieur les différents types de tranchées pour que leurs visiteurs puissent y circuler $^{78}$. À défaut d'une reconstitution complète, Ypres l'évoque grâce à un passage entre deux sections muséographiques, constitué du sol d'une tranchée protégée par une vitrine sur lequel marche le visiteur inondé d'une lumière rouge (fig. 12). À Meaux, les limites de la tranchée reconstituée sont symboliquement en blanc, couleur du mur (voir fig. 6) : des cartels précisent les différences entre les techniques utilisées par les différentes armées. Dans d'autres musées, comme à Dresde, Bruxelles et Paris, les tranchées sont présentées grâce à des maquettes ${ }^{79}$, et leurs ambiances, grâce à des agrandissements de photographies d'époque. Peu de tirages photographiques originaux sont utilisés dans les parcours muséographiques traitant de la Grande Guerre ${ }^{80}$, sauf à Berlin et à Ypres, où un ingénieux usage scénographique de binoculaires permet aux visiteurs de découvrir des images en trois ou deux dimensions. 
Figure 12

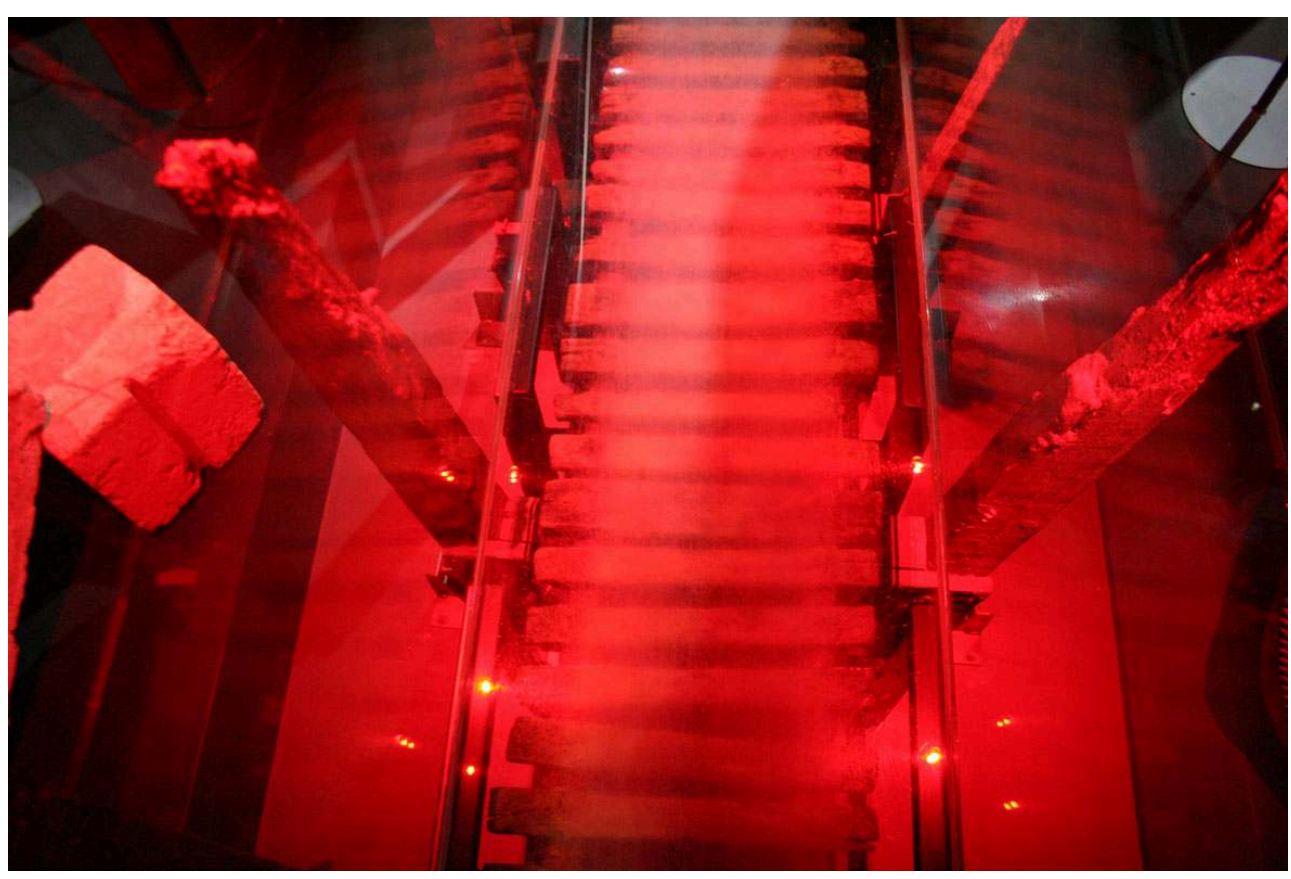

Traverses de tranchée protégées par une vitrine sur laquelle le visiteur peut marcher. In Flanders Fields Museum, Ypres.

Phot. Fleury, Célia, décembre 2013. @ Célia Fleury.

Pour évoquer la guerre sous tous les fronts, terre, mer et ciel, les musées doivent utiliser de manière optimale leurs espaces d'exposition contraints. Si Londres et Bruxelles peuvent mettre en valeur des avions et des véhicules dans de grands espaces, hall d'accueil pour le premier (voir fig. 5), et clôture en cul-de-sac de l'ancienne section Grande Guerre pour le second, les autres utilisent des maquettes ou des objetssynecdoques, comme une hélice d'avion à Dresde, Ypres et Paris (fig. 13), ou comme une cloche de navire à Dresde et Berlin (voir fig. 7). L'exposition en plein air n'existe qu'à Çanakkale, qui montre, par exemple, des fragments d'un sous-marin allemand retrouvé dans le détroit des Dardanelles. Les photographies aériennes réalisées par des aviateurs britanniques et allemands sont utilisées à Ypres pour appréhender le territoire vu du ciel et repérer les impacts de la guerre sur le terrain (disparition d'habitations, trous d'obus, tranchées, etc.) : le travail de documentation exhaustif, réalisé par le musée pour la muséographie actuelle, permet au visiteur de visualiser, à plusieurs années d'intervalle, les zones couvertes par ces photographies, grâce à une table interactive. Le lien avec le territoire actuel est aussi créé grâce à des points de vue élevés permettant l'accès à des panoramas extérieurs: au milieu du parcours muséographique, le visiteur peut faire l'ascension du beffroi à Ypres ; l'architecture du dernier étage de Dresde permet aussi une vue sur la ville, comme à Meaux sur le paysage environnant. L'interprétation de la Grande Guerre "dans tous ses états » dépasse les parcours muséographiques pour inviter le visiteur à arpenter les lieux de guerre, parfois devenus mémoriels ${ }^{81}$. 


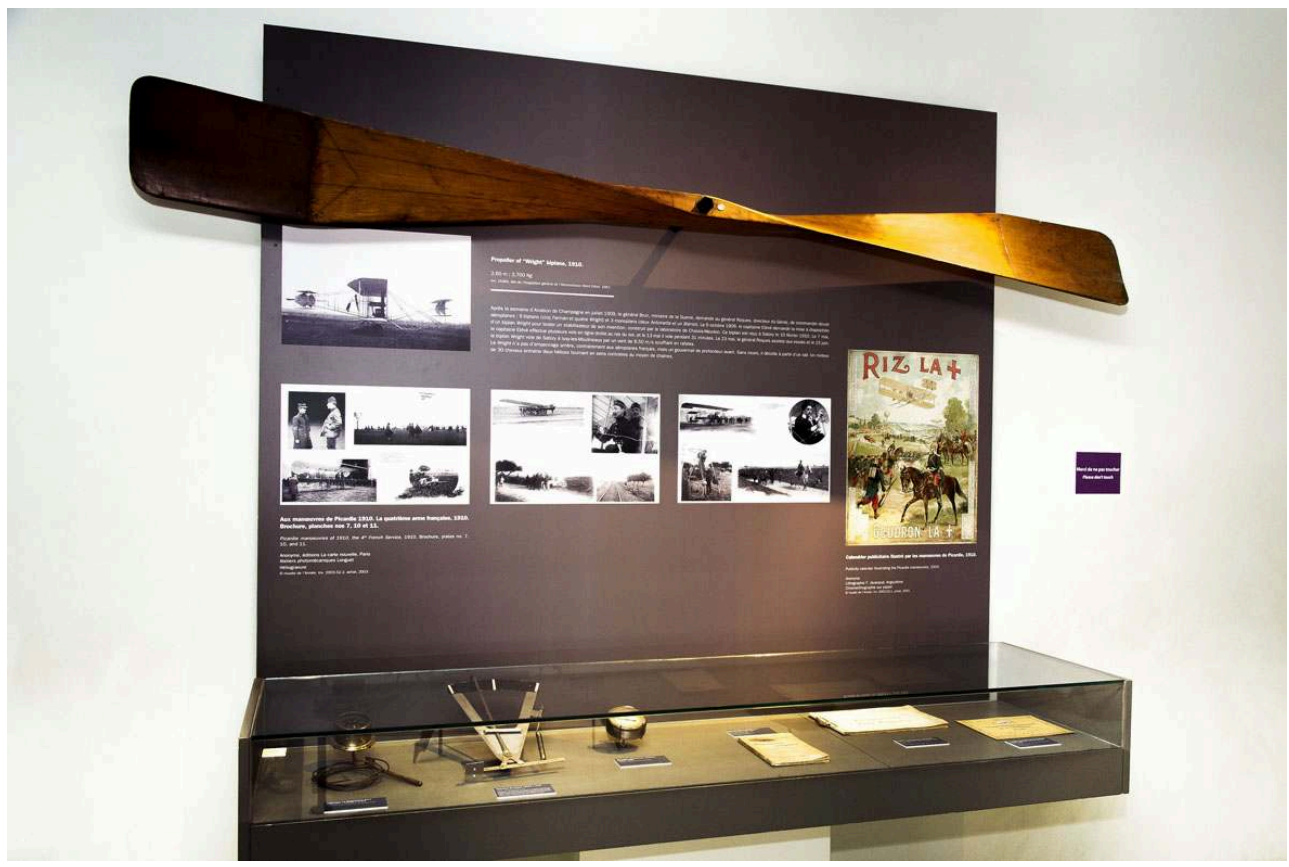

Hélice d'un biplan de l'armée française. Espaces « Première Guerre mondiale ». Musée de l'armée, Hôtel national des Invalides, Paris.

Phot. Marre-Noël, Anne-Sylvaine. (c) Paris, musée de l'Armée, dist. RMN-GP/.

\section{Les « cultures de guerre »}

Pour traiter dans les parcours des «cultures de guerre $»^{82}$, qui visent à comprendre l'engagement de l'arrière et des civils et leurs liens indirects avec le front, les musées disposent de plusieurs types d'objets : très révélateurs de l'emprise symbolique des faits de guerre sur la vie quotidienne des civils, sont les jeux pour enfants ${ }^{83}$, adaptés aux nouveaux enjeux du conflit mondial. Par exemple, la bataille navale, opposant navires à flot et sous-marins, fait son apparition, devenant ainsi l'un des jeux les plus populaires $\mathrm{du} \mathrm{Xx}^{\mathrm{e}}$ siècle. Ce thème est donc récurrent à Berlin, Ypres et Meaux. De plus, le musée de la Poupée et du Jouet ancien de Wambrechies (Nord) a même réalisé un parcours complet retraçant les grands événements de la Grande Guerre grâce uniquement à des jouets, pas nécessairement contemporains des événements, car les entreprises qui en fabriquaient étaient aussi réquisitionnées ${ }^{84}$. Ainsi, la transformation progressive des productions pour servir à l'industrie d'armement est montrée à Berlin et Dresde par une série d'objets métalliques en transformation, du plat au casque. L'emploi des femmes dans les entreprises est largement évoqué à Londres et Dresde, ce dernier musée le traitant dans sa galerie thématique commune aux deux guerres mondiales. Par leur destination et leurs modes d'accrochage originels, les affiches de propagande sont présentes dans tous les musées du corpus (fig. 14). En revanche, les problèmes de conservation préventive du papier, très sensible à la lumière et dont l'acidité le fait jaunir et devenir cassant, ont obligé certains musées à ne présenter que des fac-similés, leur permettant de créer artificiellement un mur d'affiches collées comme à Meaux. Les affiches exposées traitent toujours des mêmes thèmes, la diabolisation de l'ennemi, les souscriptions publiques pour financer la guerre ${ }^{85}$ et le recrutement de volontaires dans les pays anglo-saxons ${ }^{86}$. Çanakkale en vient même à détourner l'affiche "Your country 
needs you $\|^{87}$ comme logo implicateur, introduisant la traduction anglophone des panneaux de salle.

Figure 14

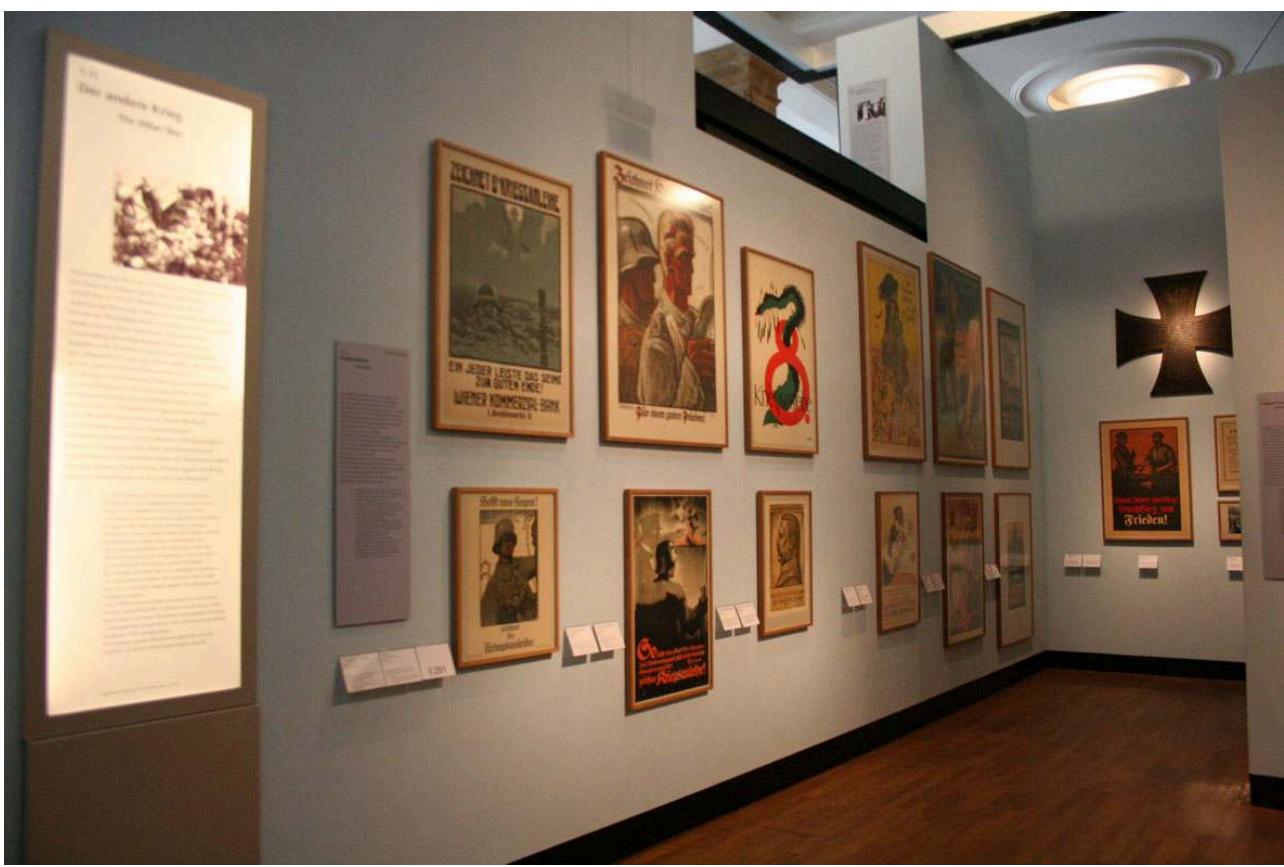

Affiches de propagande pendant la Grande Guerre et croix clouée. Deutsches Historisches Museum, Berlin.

Phot. Fleury, Célia, mai 2013. @ Célia Fleury.

Le fossé entre la propagande officielle et les conditions de vie des soldats est perceptible grâce à de nombreux objets leur ayant appartenu, souvent donnés aux musées par les familles. Si l'historiographie anglo-saxonne est la première à avoir donné une place à l'individu, certaines vitrines de Londres montrent des accumulations d'objets personnels et comme à Çanakkale, Dresde utilise l'accumulation de photos individuelles (voir fig. 2) et Paris expose la veste d'un uniforme pleine de boue. Amplifiée dans la muséographie actuelle, Ypres donne une place importante aux parcours de vie individualisés : en parallèle de la personne réelle correspondant le mieux à ses spécificités (sexe, nationalité) que le musée propose au visiteur de suivre tout au long de son parcours, sont visibles des vitrines dédiées à différentes personnes ayant laissé des traces personnelles du conflit, par exemple Rudolf Lange (voir fig. 9) ${ }^{88}$. D'autres objets témoins sont presque des reliques, comme les fragments d'Ypres et de sa cathédrale, revenus a posteriori dans la ville ou conservés partout où le don a pu être possible. Le fragment et la perte deviennent particulièrement évocateurs de la destruction totale : à Ypres, l'une des dernières sections du parcours est consacré à la reconstruction complète de la ville à l'identique dans les années 1920. Au-delà des objets, les musées essayent de muséifier le témoignage oral : à Çanakkale, un diorama expose deux vieillards qui représentent deux soldats ayant réellement témoigné à la fin de leur vie (voir fig. 1), tandis qu'Ypres oralise des témoignages écrits grâce à la performance d'acteurs, par exemple pour évoquer la trêve de Noël 1914 vécue par des soldats de différentes nationalités (fig. 15). Le choix muséographique a été de faire 
parler ces témoignages dans leurs langues originelles, sous-titrées dans les trois autres langues.

Figure 15

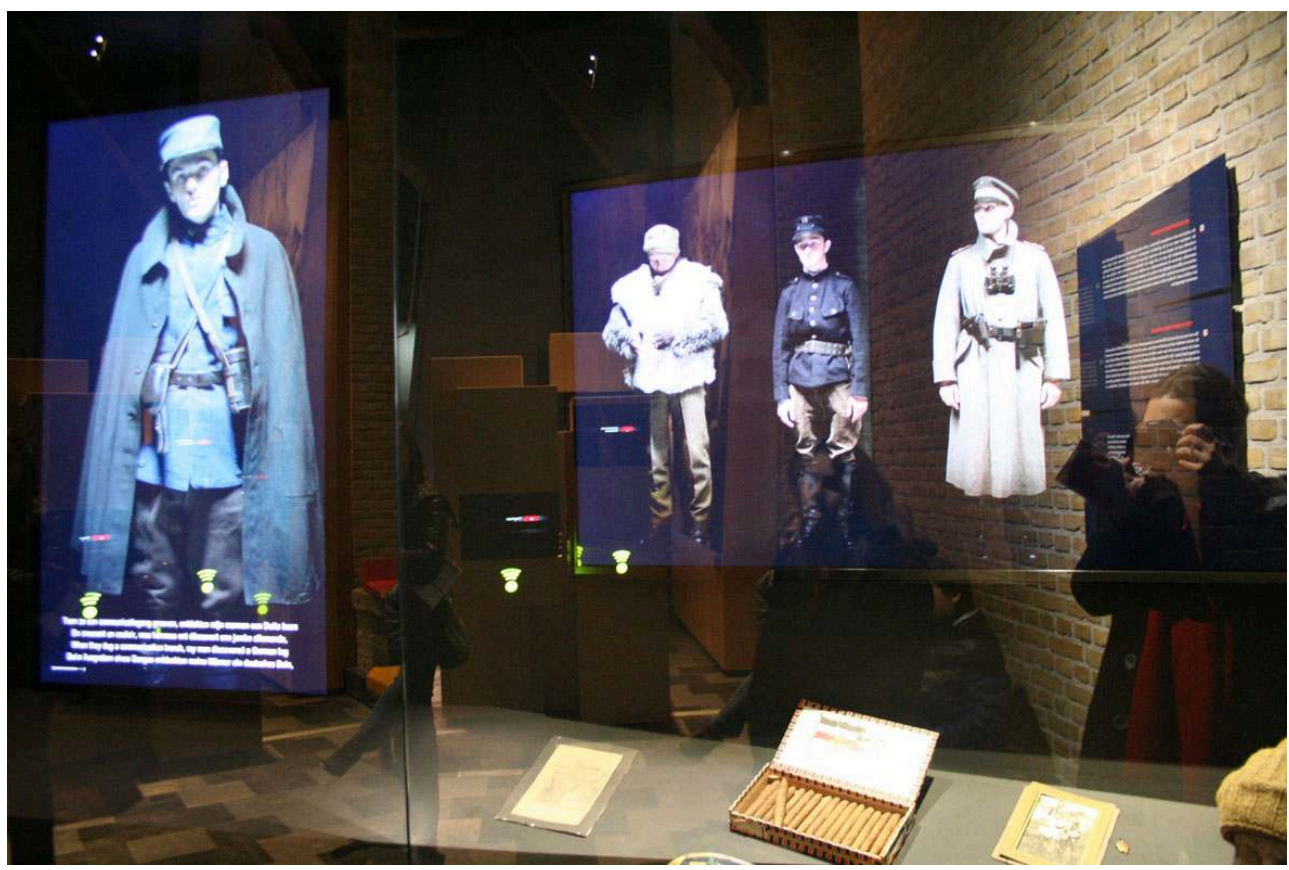

Vitrine sur la trêve de Noël 1914 : objets et témoignages authentiques, joués par des acteurs. In Flanders Fields Museum, Ypres.

Phot. Fleury, Célia, décembre 2013. @ Célia Fleury.

\section{Des interprétations différenciées de certains aspects de la Grande Guerre}

Les traditions historiographiques nationales se révèlent pleinement dans les muséographies traitant de l'internationalisation du conflit. Si l'entrée en guerre des États-Unis en 1917 fait l'objet d'une vitrine autonome à Berlin, Dresde et Londres, l'implication des ANZAC dans l'armée britannique et des troupes coloniales dans l'armée française est traitée de manière différenciée en fonction des territoires et de leurs perceptions. À Çanakkale, les soldats australiens font l'objet de vitrines spécifiques, alors que les Britanniques et les Français sont juste évoqués: le centre d'interprétation de Gallipoli (voir fig. 3) insiste même paradoxalement sur la comparaison entre le courage des soldats australiens et ottomans. Si l'enrôlement et l'intégration des troupes coloniales dans l'armée française fait l'objet d'une historiographie française relativement récente, ce thème est traité depuis les origines par l'historiographie allemande. En effet, largement utilisée par la propagande allemande pour dénoncer la barbarie française, la place des troupes coloniales est largement traitée par les muséographies allemandes, notamment dans l'entre-deuxguerres quand ces dernières participent à l'occupation de la Ruhr : à Berlin, plusieurs affiches de propagande contre l'occupation montrent le soldat français sous les traits exclusifs d'un «tirailleur sénégalais » (fig. 16). Il faut attendre 2003 pour que ce sujet fasse l'objet d'une thèse française ${ }^{89}$ : ce thème, comme les objets pouvant l'illustrer, 
n'est donc pas présent dans les muséographies françaises, même si les uniformes de soldats des colonies pendant la Grande Guerre sont désormais bien exposés pour montrer l'implication de l'Afrique noire française, mais aussi du Maghreb.

Figure 16

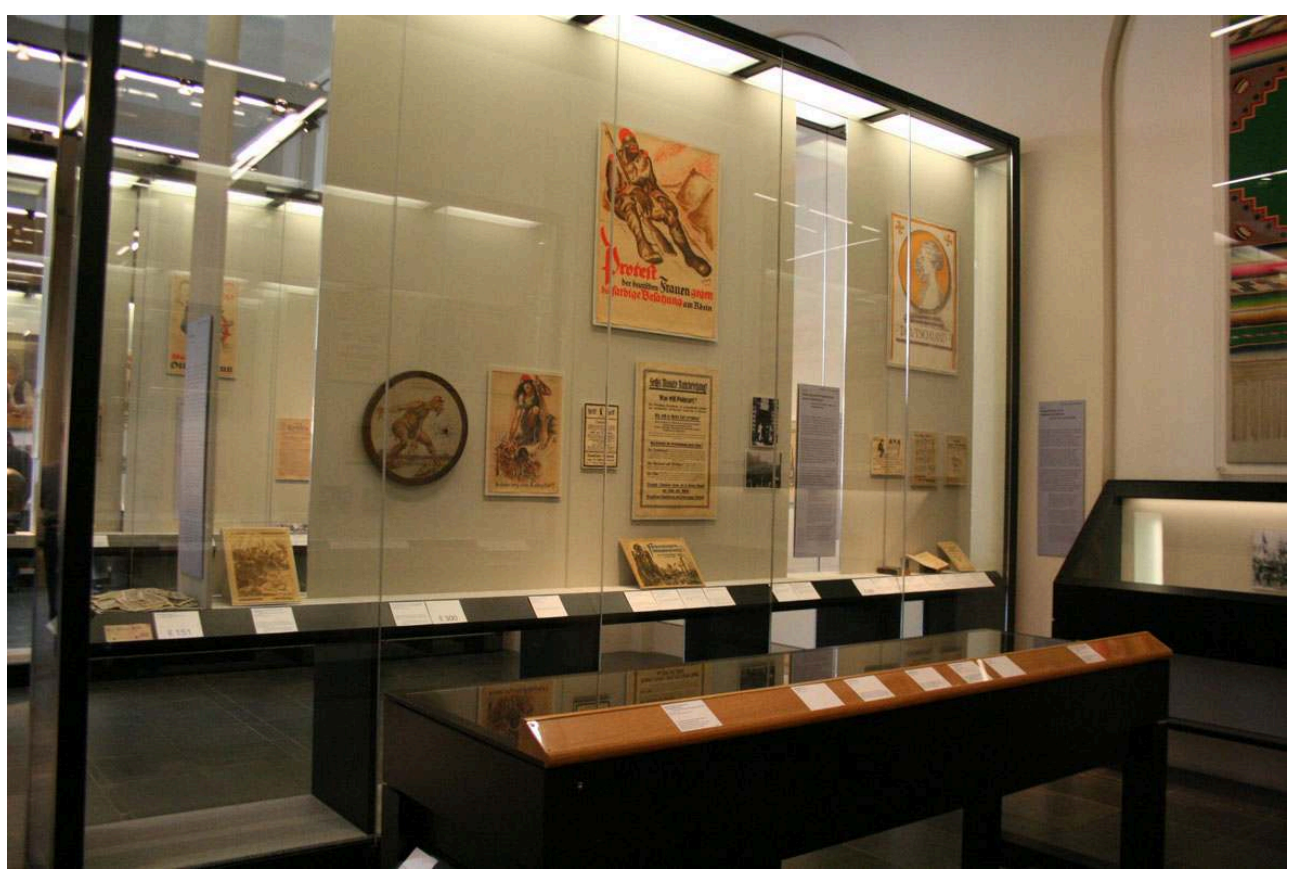

Espace traitant l'occupation de la Ruhr. Deutsches Historisches Museum, Berlin.

Phot. Fleury, Célia, mai 2013. (c) Célia Fleury.

Le traitement muséographique de la République de Weimar est un autre exemple frappant des perceptions historiques et muséographiques différenciées de la fin de la Grande Guerre. «Dans l'Allemagne de Weimar en particulier, la difficulté d'accepter la défaite n'introduisit pas seulement des procès pour détournement : l'opposition entres visions militaristes et pacifistes de l'expérience de guerre se traduisit dans des choix pleins de significations symboliques » : cette analyse de Massimo Baioni concernant la création de musées en Allemagne dans l'après-guerre, par exemple Zeughaus et Antikriegsmuseum à Berlin, semble toujours être d'actualitée ${ }^{90}$. Si Berlin traite conjointement, dans un même panneau de salle "1918-1919. Kriegsende und Revolution " (la fin de la guerre et la révolution), les révolutions russes et allemandes de 1917 et de 1918, Dresde fait le choix de ne pas traiter du tout cette période historique : une galerie thématique fait en effet le lien entre les deux conflits mondiaux (voir fig. 10). De même, alors que dans l'historiographie française, la République de Weimar est plutôt perçue comme un essai avorté entre les deux Reich, Berlin la traite comme la première tentative démocratique en Allemagne: le panneau de salle est intitulé «1918-1925. Die schweren Anfänge der Republik» (les débuts difficiles de la République). Par ailleurs, la dureté du traité de Versailles vis-à-vis des vaincus est présentée de manière homogène dans les parcours français et allemands (fig. 17), Berlin insistant même sur les rapprochements entre les deux ministres des Affaires étrangères, Aristide Briand et Gustav Streseman, dès 1925, dans ses ateliers pédagogiques. Les liens directs entre les crises politiques et financières (dévaluation 
monétaire) et l'avènement des totalitarismes sont d'ailleurs largement traités à Berlin et Bruxelles.

Figure 17

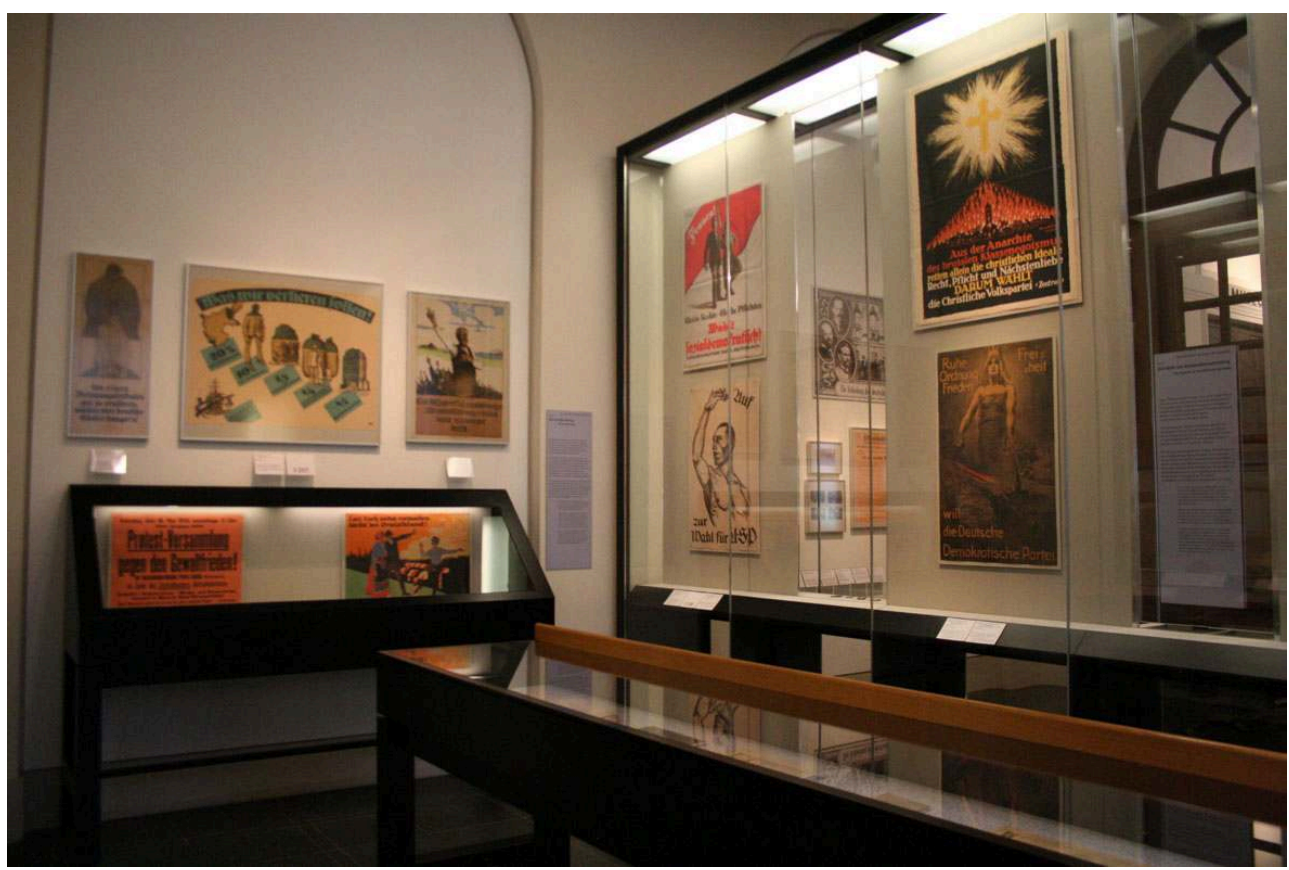

Espace consacré au traité de Versailles. Deutsches Historisches Museum, Berlin.

Phot. Fleury, Célia, mai 2013. @ Célia Fleury.

Les musées territoriaux, n'ayant pas vocation à traiter l'entre-deux-guerres, insistent davantage sur la déshumanisation de la guerre, relayée à la fin de leur parcours par le retour des soldats mutilés, les restes humains régulièrement exhumés sur les lieux de bataille, et les nombreux monuments aux morts (voir fig. 7), cimetières et mémoriaux qui scandent le paysage. Les effets directs et indirects du conflit sur les corps restent difficilement muséographiables: dans sa nouvelle muséographie ${ }^{91}$, Ypres accentue les liens physiques entre port du masque à gaz et déshumanisation de la guerre (fig. 18). 
Figure 18

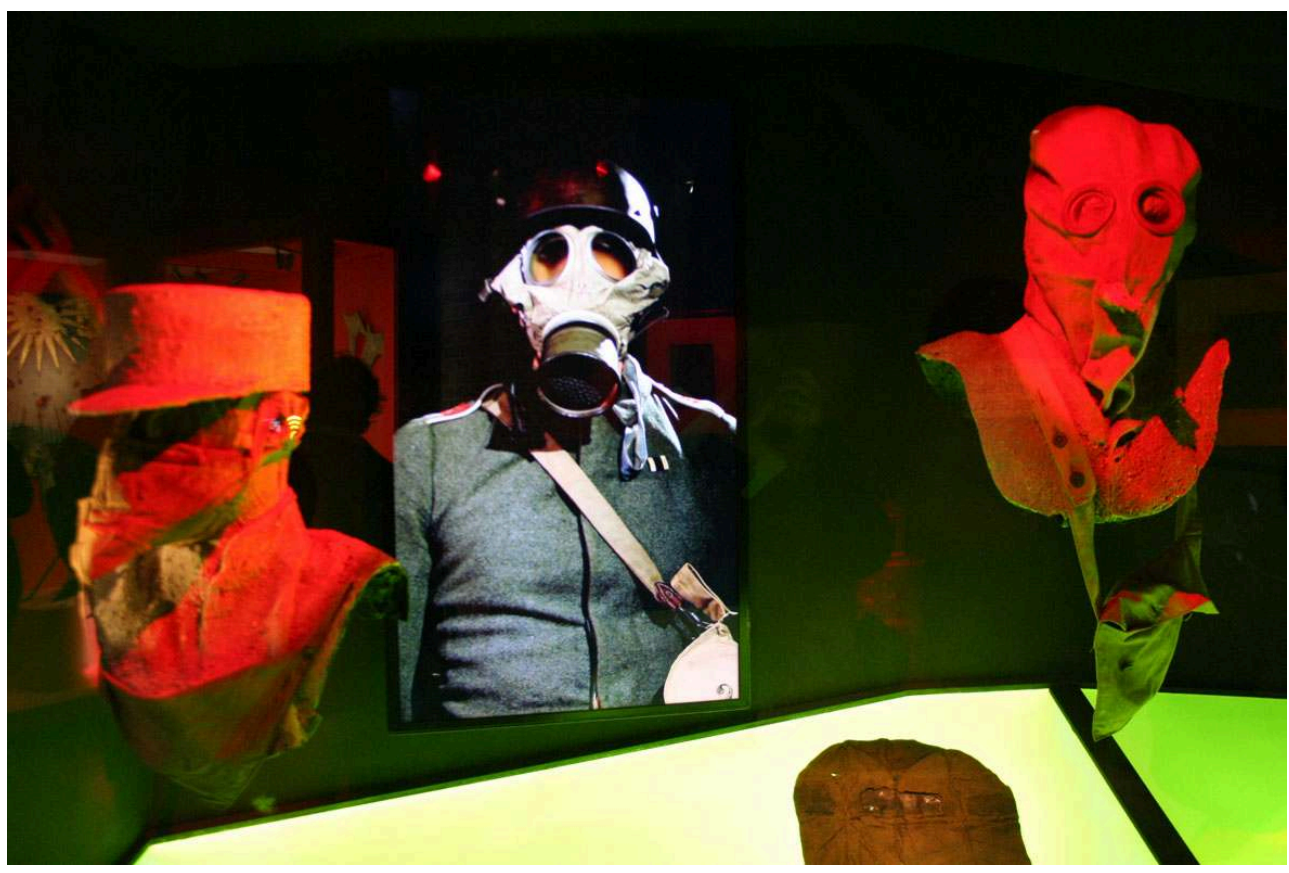

Vitrine traitant du port du masque à gaz, en associant des objets authentiques et des films montrant des soldats de chaque nationalité recouvrant leurs visages. In Flanders Fields Museum, Ypres.

Phot. Fleury, Célia, décembre 2013. ㄷ Célia Fleury.

À Ypres, le rôle de la Croix-Rouge dans la prise en charge des blessés est moins présent depuis 2012, mais le reste symboliquement à Péronne, ainsi qu'à Berlin et Dresde par l'exposition d'objets cloués (voir fig. 14) ${ }^{92}$. De plus, des espaces à part, noirs, sous forme de tentes pyramidales en dur à Ypres et de grands cubes à Dresde, permettent de signifier aux visiteurs qu'ils entrent dans un lieu à statut spécifique : ils tentent de scénariser l'horreur des restes humains fragmentés ou des " gueules cassées " $^{93}$. Jusqu'à 2011, les photographies de soldats défigurés à cause du combat étaient peu montrées : en 1988 encore, certains visiteurs s'étaient indignés lors de la préfiguration de l'Historial de la Grande Guerre à Péronne ${ }^{94}$. À Meaux, il n'y a quasiment plus aucune distance scénographique : la vitrine explicitant les "gueules cassées» est située au milieu du parcours thématique, tandis que le visiteur sort en marchant sur des photographies de restes humains exhumés. Ypres est le seul musée à terminer son parcours sur une liste de guerres qui ont eu lieu après la Grande Guerre, semblant ainsi signifier que la paix n'est jamais acquise malgré tous les sacrifices humains (fig. 19). 
Figure 19

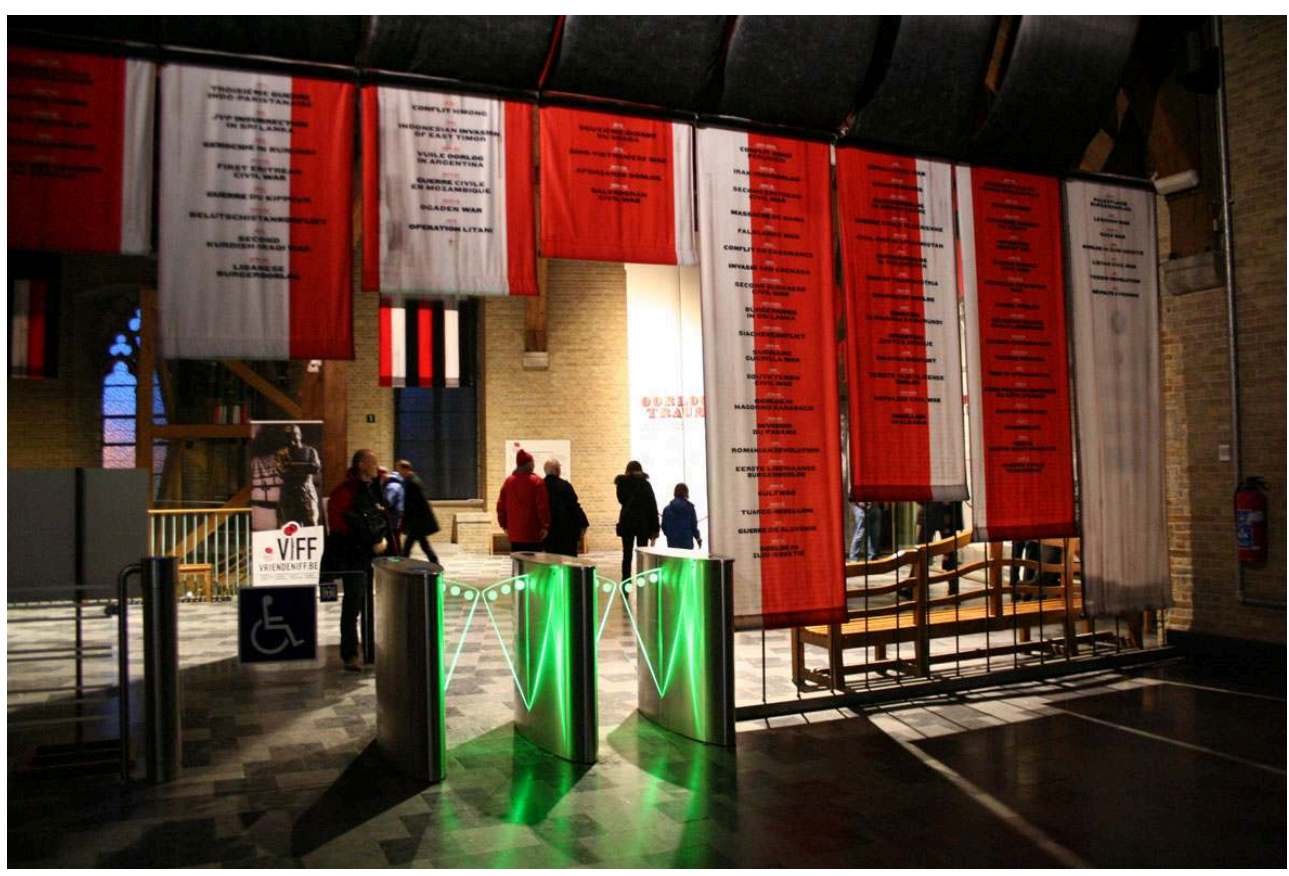

Sortie de l'exposition permanente. In Flanders Fields Museum, Ypres.

Phot. Fleury, Célia, décembre 2013. ㄷ Célia Fleury.

30 Cet état des lieux des muséographies de la Grande Guerre dans les principaux musées de l'Europe occidentale en 2013 montre que les musées sont en interaction constante avec leurs contemporains et tendent aussi à évoluer avec eux : lesquels influencent les autres? Antoine Prost et Jay Winter concluent

[en proposant d']accepter la pluralité irréductible des histoires [...] [et de] faire triompher la raison et la volonté d'intelligence sur les sentiments auxquels l'historien n'échappe pas plus que quiconque, et encore moins sur un sujet comme la guerre. Ce n'est pas s'astreindre à une écriture impersonnelle ou insensible, mais rechercher la justesse et la vérité ${ }^{95}$.

31 Probablement le concepteur de parcours muséographiques sur la Grande Guerre veut-il aussi faire preuve de sincérité et d'humilité en essayant de proposer une analyse et un récit d'un événement complexe et inédit, majeur, $\mathrm{du} \mathrm{xx}^{\mathrm{e}}$ siècle, avec le temps et les moyens dont il dispose. La transdisciplinarité (histoire, archéologie, anthropologie, muséologie, etc.) est nécessaire et des sources restent inexploitées, par exemple les photographies, notamment en couleur, qui étaient encore récemment sous droit d'auteur ${ }^{96}$, ou celles liées à l'occupation allemande du nord de la France ${ }^{97}$. 2013, l'année qui précède le centenaire constituait un cap, avec l'ouverture de musées qui proposaient une nouvelle muséographie (Dresde, Meaux, Ypres) et d'autres qui restructuraient les leurs (Londres, Péronne) au risque peut-être de les uniformiser : les visiteurs de 2014-2018 continueront à venir dans ces musées avec des attentes toujours renouvelées, faisant délibérément de ces musées des lieux du temps présent aux interprétations complémentaires où la question du "patrimoine sensible ", pouvant susciter de fortes réactions, tente d'être traitée dans toutes ses dimensions nationales, historiques et symboliques ${ }^{98}$.

«L'historien doit accepter ses limites, ou plutôt les limites du discours historique : il est des seuils qu'il ne doit pas franchir, des silences qu'il ne doit pas rompre ${ }^{99}$. À côté de lui, 
au-delà de son discours, la place reste largement ouverte pour la poésie et la médiation $»^{100}$.

\section{NOTES}

1. - HORNE, John. "Conclusion: La Grande Guerre dans l'histoire ». Dans AUDOIN-ROUZEAU, Stéphane, BECKER, Jean-Jacques (dir.). Encyclopédie de la Grande Guerre 1914-1918. Histoire et culture. Paris : Perrin, 2004, p. 946.

2. - Sur musées et lieux de mémoire, voir BRANDT, Suzanne. "The Memory Makers : Museums and Exhibitions of the First World War ». History and Memory, 1994, n¹, p. 95-122 ; HEIMBERG, Charles (dir.). « Musées, histoire et mémoires ». Le cartable de Clio. Revue suisse sur les didactiques de l'histoire, 2011, n¹1; « Historial, musées et mémoriaux de la Grande Guerre ». Guerres mondiales et conflits contemporains, 2009, n²35, p.3-85; KAVANAGH, Gaynor. Dream Spaces: Memory and the Museum. Londres : Leicester University Press, 2000 ; PÉNICAUT, Emmanuel, TOSCANO, Gennaro (dir.). Lieux de mémoire, musées d'histoire. Paris: La Documentation Française, 2012 ; POULOT, Dominique. "Musées et guerres de mémoires: pédagogie et frustration mémorielle». Dans BLANCHARD, Pascal, VEYRAT-MASSON, Isabelle (dir.). Les Guerres de mémoires. La France et son histoire. Enjeux politiques, controverses historiques, stratégies médiatiques. Paris : La Découverte, 2008, p. 230-240 ; SHERMAN, Daniel J. «Objects of memory: history and narrative in French war museums ». French Historical Studies, 1995, vol. 19, n¹, p. 49-74.

3. - THIEMEYER, Thomas. Fortsetzung des Krieges mit anderen Mitteln, Die beiden Weltkriege im Museum. Paderborn [u. a.] : Ferdinand Schöningh, 2010, p. 21.

4. - Terme assez courant en muséologie pour désigner n'importe quel objet exposé, y compris non patrimonial.

5. - FLEURY, Célia. «L'expérience des visiteurs dans les musées de la Grande Guerre : quelques pistes pour le dialogisme ». ICOFOM Study Series, 2011, n40, Paris-Taipei, p. 75-85: voir le site http://network.icom.museum/icofom/publications/our-publications/>.

6. - Sur l'apport de la redécouverte d'objets de la Grande Guerre : CORNISH, Paul, SAUNDERS, Nicholas J. (dir.). Contested Objets. Material Memories of the Great War. Londres : Routledge, 2009.

7. - Serge Chaumier montre comment il est difficile a posteriori de reconstituer le scénario originel d'une exposition (Traité d'expologie. Les écritures de l'exposition. Paris : La Documentation Française, 2012, p. 28). Et les documents de préparation, quand ils sont conservés et accessibles, ne sont qu'une vision théorique d'un parcours muséographique optimal: le conservateur de musée sait que des modifications en cours de réalisation sont nécessaires pour s'adapter aux différentes contraintes (lieu, objets, etc.).

8. - BAIONI, Massimo. "Commémorations et musées». Dans AUDOIN-ROUZEAU, Stéphane, BECKER, Jean-Jacques (dir.). Encyclopédie de la Grande Guerre 1914-1918. Histoire et culture. Paris : Perrin, 2004, p. 809.

9. - CHAUMIER, Serge, op. cit., p. 23, 27 et 28.

10. - CHAUMIER, Serge, op. cit., p. 55.

11. - THIEMEYER, Thomas, op. cit., p. 35.

12. - Concept utilisé dans plusieurs publications, il ne recouvre pas, à mon sens, la complexité d'un projet muséographique (commanditaire, enjeux scientifiques, perception potentielle des publics, financements, etc.), qui ne peut aboutir que grâce à des équipes pluridisciplinaires 
(BAIONI, Massimo, op. cit., p. 805). Comme n'importe quels autres publications ou media, l'exposition peut être le résultat, ou non, d'un travail méthodologiquement sincère. $\mathrm{S}$. Wahnich reconnait que « les outils de la critique restent à la fois faibles au regard de 'l'institution musée' si complexe à maîtriser, et éclatés selon que cette critique émane des historiens, des politiques, des archivistes ou conservateurs, ou encore des muséographes, des scénographes. " ( Réfléchir l'histoire des guerres au musée ». Culture et musées, janvier 2013, n²0, p. 13).

13. - Notamment, l'historiographie russe n'a pas pu être intégrée (PROST, Antoine, WINTER, Jay. Penser la Grande Guerre. Un essai d'historiographie. Paris: Éditions du Seuil, 2004, p. 13). T. Thiemeyer mentionne aussi la barrière linguistique (op. cit., p. 23).

14. - Grâce au séjour professionnel proposé par la direction générale des patrimoines (ministère de la Culture et de la Communication), la Stiftung Haus der Geschichte der Bundesrepublik Deutschland et l'office Franco-Allemand pour la Jeunesse, j'ai été accueillie pendant deux mois par le Deutsches Historisches Museum et je tiens à remercier particulièrement le Dr. Alexander Koch, président, et Dr. Dieter Vorsteher-Seiler, directeur du département des collections, ainsi que Dr. Juliane Haubold-Stolle pour ses conseils et nos nombreux échanges.

15. - Par exemple, ce thème ne semble être que peu analysé par l'équipe de l'ANR « Les présents des passés» du laboratoire CRISES de l'université Paul-Valéry Montpellier III, pourtant constituée majoritairement d'historiens. Voir LOUVIER, Patrick, MARY, Julien, ROUSSEAU, Frédéric. Pratiquer la muséohistoire. La guerre et l'histoire au musée. Pour une visite critique. Outremont : Athéna éditions, 2012 et ROUSSEAU, Frédéric (dir.). Les présents des passés douloureux, Musées d'histoire et configurations mémorielles. Paris : Houdiard Michel Eds, 2012 ; bibliographie du projet ANR, voir le site : http://crises.upv.univ-montp3.fr/programmes-anr-et-dfg/programmepop-anr-les-presents-des-passes/ressources/bibliographie-alphabetique/.

16. - PROST, Antoine, WINTER, Jay, op. cit., p. 13.

17. - Le front est donc traditionnellement beaucoup moins analysé en Europe occidentale (voir cidessus et HORNE, John, op. cit., p. 938). John Keegan précise qu'il n'y a pas d'histoires officielles de la Grande Guerre en Russie et en Turquie (The First World War. London : Hutchinson/Random House, 1998 ; trad. fr. Paris : Perrin, 2003, p. 535).

18. - Le récent ouvrage de Christopher Clark redistribue encore les responsabilités potentielles (Les somnambules. Été 14 : comment l'Europe a marché vers la guerre. Paris : Flammarion, 2014).

19. - PROST, Antoine, WINTER, Jay, op. cit., p. 268-270.

20. - P. Nora (Les Lieux de mémoire. Paris: Gallimard, 1984-1992, 3 tomes) est cité dès 1994 : GRÜTTER, Heinrich Theodor. «Die Präsentation der Vergangenheit. Zur Darstellung von Geschichte in historischen Museem und Ausstellungen ». Dans FÜSSMANN, Klaus, GRÜTTER, Heinrich Theodor (dir.). Historische Faszination. Geschichtskultur heute. 1994, Köln-Weimar-Wien, Böhlau Verlag, p. 174.

21. - Voir notamment, KORTE, Barbara, PALETSCHEK, Sylvia, HOCHBRUCK, Wolfgang. Der Erste Weltkrieg in der populären Erinnerungskultur. Essen : Klartext Verlag, 2008 ; THIEMEYER, Thomas, op. cit., 2010, p. 77-93.

22. - Pour M. Baioni «l'étude de la mémoire de la Grande Guerre (...) un des laboratoires privilégiés du dialogue interdisciplinaire » (op. cit., p. 804).

23. - Voir la difficile synthèse franco-allemande sur l'occupation de la Ruhr, évoquée plus bas.

24. - Pour M. Baioni, les musées ont probablement un rôle important dans la constitution d'un « imaginaire de guerre », même si les données concernant la réception manquent (op. cit., p. 811). 25. - BAIONI, Massimo, op. cit., p. 802 et 803.

26. - LONDEY, Peter, STEEL, Nigel. «Der Erste Weltkrieg als nationaler Erinnungsort. Das 'Imperial War Museum' in London und das 'Australian War Memorial' in Canberra ». Dans KORTE, Barbara, PALETSCHEK, Sylvia, HOCHBRUCK, Wolfgang (dir.). Der Erste Weltkrieg in der populären Erinnerungskultur, 2008. Essen : Klartext Verlag, p. 42-43. 
27. - KENNAN, George F. The Decline of Bismarck's European Order. Franco-Russian Relations, 1875-1890. Princeton : Princeton University Press, 1979, p. 3. Ce concept est, par exemple, mentionné par T. Thiemeyer (op. cit., p. 21).

28. - SCATES, Bruce. Return to Gallipoli. Walking the Battlefields of the Great War. Cambridge: Cambridge University Press, 2006 ; BAIONI Massimo, op. cit., p. 802.

29. - Voir PROST, Antoine, WINTER, Jay, op. cit., p. 287 ; WAHNICH, Sophie. «Trois musées de guerre $\mathrm{du} \mathrm{xx}^{\mathrm{e}}$ siècle: Imperial War Museum de Londres, historial de Péronne, mémorial de Caen ». Dans BOURSIER, Jean-Yves. Musées de guerre et mémoriaux. Paris : Maison des Sciences de l'Homme, 2005, p. 65-81, voir le site : http://books.openedition.org/editionsmsh/958, (\$11).

30. - Cette étude (Planeth-Département du Nord, 2013) compare les programmes scolaires nationaux, qui reflètent aussi les différentes traditions historiographiques : par exemple, si les batailles d'Ypres sont traitées dans les programmes belges, hollandais et canadiens, Verdun reste une spécificité française. La guerre de tranchées est présente au Royaume-Uni, en France et en Allemagne ; la guerre totale et l'économie de guerre, en France, Belgique et Allemagne.

31. - Par exemple, les galeries «Great War » de l'Australian War Memorial.

32. - S. Wahnich qualifie l'IWM Londres comme un lieu de "rêverie glorieuse, individuelle et collective", sans vraiment analyser les liens intrinsèques entre l'historiographie et la muséographie anglo-saxonne (op. cit., § 11-15). Si M. Baioni évoque clairement son contexte de création, mais faute d'études sur la réception des différents types de visiteurs (étudiants, militaires, vétérans, associations, simples citoyens, etc.), il ne peut analyser les raisons de la fréquentation massive (op. cit., p. 808 et 811). C. Beil se pose la question des frontières entre la banalisation de la guerre et de la violence et le mauvais goût (« Musealisierte Gewalt. Einige Gedanken über Präsentationsweisen von Krieg und Gewalt in Austellungen ». Museumskunde, 2003, H. 1, 68, p. 7-17).

33. - La bibliographie sur les musées d'histoire est d'ailleurs davantage franco-allemande, grâce notamment aux travaux de l'Association Internationale des Musées d'Histoire (AIMH) et la Haus der Geschichte de Bonn : voir, notamment, GERVEREAU, Laurent (dir.). Quel avenir pour les musées d'histoire? AIMH-Ministère de la Culture-Haus der Geschichte, 1999, et JOLY, Marie-Hélène, COMPÈRE-MOREL, Thomas (dir.). Des musées d'histoire pour l'avenir. Paris : Noêsis, 1998.

34. - HINZ, Hans-Martin (dir.). Der Krieg und seine Museen. Für das Deutsche Historische Museum herausgegeben von Hans-Martin Hinz. Frankfurt/New York : Campus Verlag, 1997.

35. - BEIL, Christine. Der ausgestellte Krieg. Präsentationen des Ersten Weltkriegs 1914-1939. Tübingen : Tübingen Vereinigung für Volkskunde, 2004.

36. - THIEMEYER, Thomas. Fortsetzung des Krieges mit anderen Mitteln, op. cit. ; « Zwischen Helden, Tätern und Opfern. Welchen Sinn deutsche, französische und englische Museen heute in den beiden Weltkriegen sehen ». Geschichte und Gessellschaft, 2010, p. 462-491. En anglais « Exhibiting the War. The Meaning of the Two World Wars Today in German, French, and British Museums ». Dans SCHEER, Monique (Ed.). Out of the Tower. Tübingen : Tübinger Vereinigung für Volkskunde, 2013, p. 288-307.

37. - Voir aussi BEIR-DE-HAAN, Rosmarie. Erinnerte Geschichte - Inszenierte Geschichte. Austellungen und Museen in der Zweiten Moderne. Frankfurt am Main : Suhrkamp, 2005 ; ZWACH, Eva. Deutsche und englische Militärmuseen im 20. Jahrundert. Eine Kulturgeschichtliche Analyse des gesellschaftlichen Umgangs mit Krieg. Münster : Lit Verlag, 1999 (qui traite notamment de Dresde et Londres).

38. - Pour cet auteur (p. 22), son corpus est constitué de 10 musées militaires (dont 7 traitant de la Grande Guerre : Londres, Manchester, Paris, Péronne, Ypres, Ingolstadt et Dresde) et d'un seul musée historique (Berlin).

39. - Arsenal und Museum. Vergangenheit, Gegenwart, Zukunft, Militärhistorisches Museum der Bundeswehr, Dresden 5, 2003 ; Austellungsstücke und ihre Geschichte, Militärhistorisches Museum der Bundeswehr, Dresden 2, 2001; 100 Jahre Museum im Dresdner Arsenal (1897-1997). Eine Schrift zum Jubiläum, Dresden, 1997 ; Militär Historisches Museum Dresden, Austellungsführer. Dresde : Sandstein 
Verlag-Militärhistorisches Museum der Bundeswehr, 2011; PIEKEN, Gorch. "Contents and Space : New Concept and New Building of the Militärhistorisches Museum of the Bundeswehr ", Museum \& Society, 2012-10(3), p. 163-173, voir le site: https://www2.le.ac.uk/departments/ museumstudies/museumsociety/documents/volumes/pieken.pdf.

40. - LONDEY, Peter, STEEL, Nigel, op. cit. ; CORNISH, Paul. « Sacred Relics. Objets in the Imperial War Museum 1917-39 ». Dans CORNISH, Paul, SAUNDERS, Nicholas J. (dir.). Matters of Conflict. Materiel Culture, Memory and the First Word War. London, New York : Routledge, 2004, p. 35-50.

41. - Sur la conservation actuelle d'une partie de la muséographie de 1923 : MALO, Laurent. « La Salle Historique au musée royal de l'Armée de Bruxelles : histoire et évolution (1923-2007)». Bulletin du Musée Royal de l'Armée et de l'Histoire Militaire. Contributions à l'Étude du Patrimoine Militaire, 2007-1, p. 50-69.

42. - BARCELLINI, Caroline. Le musée de l'Armée et la fabrique de la nation. Histoire militaire, histoire nationale et enjeux muséographiques. Paris: L'Harmattan, 2010; GUILLET, David. «Le musée de l'Armée, musée d'objets, musée d'histoire. Enjeux et conditions d'une mutation ». Dans PÉNICAUT, Emmanuel, TOSCANO, Gennaro (dir.), op. cit., p. 73-86 ; RENAUDEAU, Olivier. «Les aménagements muséographiques du Musée de l'Armée depuis 1945 ». Revue de la Société des Amis du Musée de l'Armée, 2005, 129, p. 57-70.

43. - THIEMEYER, Thomas. "Ästhetisierung des Destruktiven. Wo das Museum am Krieg scheitert ». Museumkunde, $\mathrm{n}^{\circ} 74,2009-1, \mathrm{p} .84$.

44. - BENOÎT, Isabelle. «Taire la seconde guerre mondiale. Les collections militaires dans l'Allemagne divisée ». Dans WAHNICH, Sophie (dir.). Les musées des guerres du Xxe siècle: lieux du politique? Tumultes, mai 2001, nº16, p.121-145, voir le site: http://www.cairn.info/revuetumultes-2001-1.htm.

45. - Par exemple, des expérimentations sur des armes du xix ${ }^{\mathrm{e}}$ siècle à Bruxelles permettent de faire avancer la connaissance sur leurs modalités d'usage: voir AUBAGNAC, Gilles. Risques et collections industrielles: les collections militaires contemporaines, 8 avril 2010, voir le site: http:// www.armes-ufa.com/spip.php?article527.

46. - Voir le dépliant du musée: http://www.mhmbw.de/media/documents/flyer/allgemein/ Flyer\%20Besucherinformationen\%20MHM.pdf.

47. - L'auteur allemand, Gerd Krumeich, met en exergue la neutralité politique du parcours de Paris (voir « Der Erste Weltkrieg im Museum. Das 'Historial de la Grande Guerre' in Péronne und neuere Entwicklungen in der musealen Präsentation des Ersten Weltkriegs». Dans KORTE, Barbara, PALETSCHEK, Sylvia, HOCHBRUCK, Wolfgang (dir.). Der Erste Weltkrieg in der populären Erinnerungskultur. Essen : Klartext Verlag, 2008, p. 69). Voir aussi BONNEFOI, Nadine, GIRAUDIER, Vincent. «Présentation générale du parcours ». Revue de la Société des Amis du Musée de l'Armée, 2000,120 , p. 36-47 ; DELMAS, Jean. « Le point de vue d'un membre du conseil scientifique ». Revue de la Société des Amis du Musée de l'Armée, 2000, 120, p. 27-30.

48. - Pour Péronne, voir HERTZOG, Anne. «Les musées de la Grande Guerre en Picardie et leurs territoires. La patrimonialisation de la guerre dans une région en recomposition: approche géographique d'un phénomène contemporain». Dans BOULANGER, Philippe, NIVET, Philippe (dir.). La Géographie militaire de la Picardie, du Moyen Age à nos jours. Amiens: Encrage, 2006, p. 123-139.

49. - De 1999 à 2012, leur revue néerlandophone et anglophone, In Flanders Fields Magazine, listait régulièrement les donations; un répertoire chronologique est désormais en ligne, voir le site : http://www.inflandersfields.be/fr/centre-de-sciences/appels/schenkingen-2. Des collectes sont aussi organisées, par exemple, en décembre 2013 dans le cadre Europeana 1914-1918.

50. - FONTAINE, Caroline, BECKER, Annette, AUDOIN-ROUZEAU, Stéphane, PRÉVOST-BAULT, Marie-Pascale. Les collections de l'Historial de la Grande Guerre. Paris : Somogy, 2008, p. 11-13 et p. $160-167$.

51. - Le Musée de la Grande Guerre. Pays de Meaux. Un nouveau regard 14/18. Paris : Le Cherche-Midi. 
52. - Sur l'enjeu des conditions d'accès à l'exposition permanente des musées de guerre, voir TROUCHE, Dominique. «Rendre sensible l'histoire des guerres au musée: une question d'ambiances?». Dans WAHNICH, Sophie, loc. cit., 2003, p. 172-174.

53. - BURKHARD, Asmuss. «Die Dauerausstellung des Deutschen Historischen Museums, Vorgeschichte, Kritik, Gegenkritik ». Zeitgeschiche-online, juillet 2007, p. 1-16, voir le site : http:// www.zeitgeschichte-online.de/thema/die-dauerausstellung-des-deutschen-historischen-

museums ; CHARLÉTY, Véronique. «Fragment de mémoire. Le musée historique allemand de Berlin », Politix, $1^{\text {er }}$ trimestre 1996, vol.9, n³3, p. 133-155, voir le site: http://www.persee.fr/ web/revues/home/prescript/article/polix_0295-2319_1996_num_9_33_1941; HAUBOLD-STOLLE, Juliane. "Que retient le Deutsches Historisches Museum du contemporain? ». Dans Que reste-t-il du temps présent? Collecter le contemporain dans les musées de société, 2012, p. 186-191; HINZ, HansMartin. "Museology and New National Museums of History and Culture ». ICOFOM Study Series, 2006, n³5, p.13-21, voir le site: http://network.icom.museum/icofom/publications/ourpublications/; OTTOMEYER, Hans. "Le Deutsches Historisches Museum (Musée historique allemand). Principes et buts de l'exposition permanente». Dans PÉNICAUT, Emmanuel, TOSCANO, Gennaro (dir.), op. cit., p. 145-152.

54. - Voir plus haut.

55. - Ces controverses ont néanmoins déclenché une intense réflexion et la publication d'une importante bibliographie germanophone sur les musées d'histoire (voir plus haut).

56. - Sur l'histoire du DHM, MULLER, Heinrich. Das Berliner Zeughaus. Vom Arsenal zum Museum. Berlin : Brandenburgisches Verlagshaus, 1994 ; OTTOMEYER, Hans. «Le Deutsches Historisches Museum à Berlin. Musée d'histoire nationale dans la capitale allemande ». ICOM France, la lettre du comité national français, décembre 2005, n³0, p. 16-18, voir le site : http://www.icom-musees.fr/ index.php/page/index/La-Lettre-du-Comite-national-francais-de-lICOM ; QUAAS, Gerhard, KÖNIG, André. Verluste aus dem Sammlungen des berliner Zeughauses während und nach dem zweiten Weltkrieg. Berlin: Stiftung Deutsches Historisches Museum, 2011; STÖLZL, Christoph. «Zur Situation des Deutschen Historischen Museums im Berlin ». Dans FÜSSMANN, Klaus, GRÜTTER, Heinrich Theodor (dir.), op. cit., p. 145-157 ; Wir schreiben Geschichten 1987-2012. 25 Jahre Deutsches Historisches Museum. Berlin : Deutsches Historisches Museum, 2012.

57. - Étonnant pour un conservateur de musée français, le catalogue des collections, accessible sur Internet (voir le site: http://www.dhm.de/datenbank/), mais aussi celui plus complet, réservé au personnel du musée, ne précise pas les provenances des objets, ce qui pourtant permet en général des analyses plus fines. Mais 60 \% des objets exposés ont été acquis entre 1987 et 2009 (voir OTTOMEYER, Hans, op. cit., 2012, p. 148).

58. - Voir le site internet du musée : http://www.denizmuzeleri.tsk.tr/en/cdmk/.

59. - Voir le clip de présentation : http://www.youtube.com/watch?v=EpS9G75oleY.

60. - PROST, Antoine, WINTER, Jay, op. cit., p. 13-14. Des tentatives de construction d'une histoire franco-allemande existent d'un point de vue pédagogique, comme le montre la publication du Manuel d'histoire franco-allemand. L'Europe et le monde du Congrès de Vienne à 1945. Paris : Nathan, 2008.

61. - Les principales références bibliographiques sur Péronne sont: BRANDT, Suzanne. "The Historial de la Grande Guerre in Péronne, France. A Museum at the Former First World War Battlefield». Museum international, 2004, vol.56-3, n²23, p. 46-52, voir le site: http:// unesdoc.unesco.org/ulis/museum/searchfr_advanced.html (recherche et téléchargement dans archives numériques, décembre 2013); COLARDELLE, Michel. «Objets insolites, objets ethnologiques ». Petites histoires de la grande guerre. Les objets insolites de l"Historial. Péronne, 2001, p. 11-21; DESHAYES, Sophie. «Les sciences humaines et sociales s'exposent à l'Historial de Péronne ». Culture et Musées, 2007, n 10, p. 79-95, voir le site : http://www.persee.fr/web/revues/ home/prescript/article/pumus_1766-2923_2007_num_10_1_1441; HADLEY, Frédérick. « Museen und Gedenkstätten des Ersten Weltkriegs an der Somme». Dans HIRSCHFLED, Gerhard, 
KRUMEICH, Gerd, RENZ, Irina (dir.). Die Deutschen an der Somme 1914-1918. Krieg, Besatzung, Verbrannte Erde. Essen: Klartext, 2006, p. 263-273; KRUMEICH, Gerd, op. cit.; VAN GOIDSENHOVEN, Gaëtan. "L'Historial de la Grande Guerre de Péronne, 'L'Histoire autrement' ". L'invitation au musée, $1^{\mathrm{er}}$ trimestre 2007, n¹7, p. 25-27; WAHNICH, Sophie, TISSERON, Antonin. « 'Disposer des corps' ou mettre la guerre au musée. L'historial de Péronne, un musée d'histoire européenne de la guerre de 1914-1918 ». Dans WAHNICH, Sophie (dir.), op. cit., p. 55-81.

62. - BAIONI, Massimo, op. cit., p. 799-800.

63. - Selon T. Thiemeyer, Berlin et Dresde ont défini leurs concepts originaux grâce à des commissions d'experts interdisciplinaires tandis que Paris a davantage organisé sa conception en interne («Waffen und Weltkriege im Museum. Wie sich die museale Darstellung der beiden Weltkriege und der Umgang mit militaria gewandelt hat ». Militärgeschichtliche Zeitschrift, 2010-1, p. 5).

64. - Pour des photographies de cette muséographie, voir le site: http://www.historial.org/ Musee-collection/Musee/Parcours.

65. - BRANDT, Suzanne. "Exposer la Grande Guerre. La première guerre mondiale dans les expositions en Allemagne de 1914 à nos jours». Dans BECKER, Jean-Jacques (dir.). Histoire culturelle de la Grande Guerre. Paris : Armand Colin, 2005, p. 139-155.

66. - En 2013, environ 450 histoires individuelles sont disponibles pour être au plus proche des différents profils des visiteurs.

67. - Guide du Musée. Yper : In Flanders Fields Museum, 2001; In Flanders Fields, Museum Guide. Ypres : Madoc \& In Flanders Fields Museum, 1998.

68. - Sur la place du son dans les musées des deux guerres mondiales, voir TROUCHE, Dominique. Les mises en scène de l'histoire. Approche communicationnelle des sites historiques des guerres mondiales. Paris : L'Harmattan, 2010, p. 93, 94 et 99.

69. - T. Thiemeyer analyse parfaitement toutes les dimensions de l'objet muséal (op. cit., 2010, p. 263-312). S. Chaumier le reconnaît après avoir analysé la contradiction du musée comme « lieu d'accumulation des savoirs d'un côté et lieu d'accumulation d'objets sans parole de l'autre »: «l'objet est un document» (p.65 et 67). A. Prost et J. Winter listent de «multiples objets, considérés jusque-là comme des curiosités ou des souvenirs sans importance » (op. cit., p. 46).

70. - Dominiek Dendooven l'a particulièrement bien montré lors de sa communication «Collaborer avec les collectionneurs privés : les expériences d'un conservateur de musée » au symposium international TEMUSE 14-45, voir le site : http://hal.univ-lille3.fr/hal-00836341.

71. - Pour une vue d'ensemble du double parcours, chronologique et thématique, voir le site: http://www.mhmbundeswehr.de/index.php/museumsplan\#top.

72. - Voir le site: http://fr.wikipedia.org/wiki/Mus\%C3\%A9e_juif_de_Berlin\#mediaviewer/ File:Shalechet_Berlin.jpg.

73. - Paul Williams intègre ce musée dans son corpus et analyse l'esthétique minimaliste de l'architecte Daniel Libeskind : WILLIAMS, Paul. Memorial museums. The Global Rush to Commemorate Atrocities. Oxford-New York : Berg, 2007, p. 94-95.

74. - Voir le site: http://www.museedelagrandeguerre.eu/sites/default/files/images/2012/ Salle06D.jpg.

75. - Ce parti pris muséographique a été largement commenté : WAHNICH, Sophie, op. cit. § 16-21 ; TROUCHE, Dominique, LAMBERT, Emmanuelle. «La convocation des corps dans les expographies des musées d'histoire des guerres ». Culture et Musées, 2010, n¹4, p. 127-145.

76. - Voir plus haut.

77. - DESFOSSÉS, Yves, JACQUES, Alain, PRILAUX, Gilles. L'archéologie de la Grande Guerre. Rennes : Ouest-France/INRAP, 2008.

78. - Voir les sites: http://mmp.zonnebeke.be/en/ et http://presse2013.voila.net/files/ vdn3mai2013.pdf. 
79. - Voir le site: http://www.musee-armee.fr/fileadmin/user_upload/Documents/SupportVisite-Fiches-Objets/Fiches-1914-1918/MA_fiche-objet-maquette-tranchee.pdf.

80. - Pour davantage d'informations sur la place de la photographie dans la muséographie, voir BEIL, Christine, op. cit., 2003, p. 10-11 ; THIEMEYER, Thomas, op. cit., 2010, p. 304-307.

81. - FLEURY, Célia, op. cit., p. 82-83.

82. - Voir PROST, Antoine, WINTER, Jay, op. cit., p. 217-223.

83. - Sur ce thème, voir BEIL, Christine, op. cit., 2004, p. 126 ; WAHNICH, Sophie, op. cit. § 222 ; à Péronne, voir le site: http://www.historial.org/Musee-collection/Collection/Collectionsthematiques/Les-jeux-et-jouets-de-1914-1918.

84. - Voir le site: http://www.lavoixdunord.fr/Locales/Marcq_en_Baroeul/actualite/ Secteur_Marcq_en_Baroeul/2008/08/05/article_la-grande-guerre-entre-au-musee-de-la-

po.shtml et http://www.lavoixdunord.fr/region/wambrechies-l-expo-14-18-le-jouet-s-en-va-ten-ia22b49746n1680379.

85. - Par exemple « Pour la France, versez votre or... » (1915), voir le site : http://www.museearmee.fr/fileadmin/user_upload/Documents/Support-Visite-Fiches-Objets/Fiches-1914-1918/

MA_fiche-objet-affiche-france-or.pdf.

86. - Voir BRANDT, Suzanne, op. cit., 2005, p. 153; à Péronne, voir le site: http:// www.historial.org/Musee-collection/Collection/Collections-thematiques/Les-affichesde-1914-1918.

87. - Voir le site : http://commons.wikimedia.org/wiki/File:YourCountryNeedsYou.jpg.

88. - Rudolf Lange a fait l'objet d'une exposition temporaire en 2004-2005 : «War witness Rudolf Lange ", Flanders Fields Magazine, janvier 2007, vol. 9, n¹7, p. 11.

89. - LE NAOUR, Jean-Yves. La honte noire. L'Allemagne et les troupes coloniales françaises, 1914-1945. Paris, 2003 ; BEAUPRÉ, Nicolas. Le traumatisme de la Grande Guerre 1918-1933. Villeneuve d'Ascq : Presses Universitaires du Septentrion, 2012, p.139-154 (l'ouvrage a d'abord été publié en allemand en 2009).

90. - BAIONI, Massimo, op. cit., p. 807-808 et 810-811.

91. - La muséographie jusqu'en 2011 traitait déjà de cet aspect en suspendant des masques à gaz devant des poèmes ou d'autres textes imprimés (voir THIEMEYER, Thomas, op. cit., 2010, p. 69).

92. - La Croix-Rouge avait organisé de multiples expositions en Allemagne pendant la guerre pour récolter des fonds. Chaque donateur pouvait planter un clou dans un objet en bois (BRANDT, Suzanne, op. cit., 2005, p. 142-44).

93. - Sur les "gueules cassées", voir le site: http://www.musee-armee.fr/fileadmin/ user_upload/Documents/Support-Visite-Fiches-Objets/Fiches-1914-1918/MA_fiche-objet-corpsmeurtris.pdf.

94. - S. Brandt relate en effet cette indignation (op. cit., p. 148). Sur l'impossible représentation muséographique du traumatisme du soldat, voir DELAGE, Caroline. Traumatisme de guerre et muséographie. 2008, voir le site: http://defense.ac-montpellier.fr/pdf/cercle/ TRAUMATISME\%20DE\%20GUERRE\%20ET\%20MUSEOGRAPHIE.pdf.

95. - Op. cit., p. 288-289.

96. - Par exemple, les opérateurs des autochromes des Archives de la planète d'Albert Kahn qui ont fait des reportages pendant la Grande Guerre, voir le site: http://albert-kahn.hauts-deseine.net/.

97. - L'historiographie est encore récente : BECKER, Annette. Les cicatrices rouges 1914-1918, France et Belgique occupées. Paris : A. Fayard, 2010 ; HIRSCHFELD, Gerhard, KRUMEICH, Gerd, RENZ, Irina (Hg.), op. cit. ; McPHAIL, Helen. The Long Silence. Civilian Life under the German Occupation of Northern France, 1914-1918. London-New York : I. B. Tauris, 1999.

98. - Sélectionné par le ministère de la Culture et de la Communication dans le cadre de son appel à projet "Pratiques interculturelles dans les institutions patrimoniales", 2013, TEMICS «Reconnaissance, conservation et transmission de la diversité des témoignages sur les objets du 
patrimoine sensible en contexte interculturel : pratiques collaboratives et médiation numérique en musée » vise à transposer une méthodologie de récolte de témoignages multiples sur des objets des musées des deux guerres mondiales. Voir aussi RANCIÈRE, Jacques. Le partage du sensible. Paris : La Fabrique, 2000, et WAHNHICH, Sophie, op. cit., 2013, p. 16.

99. - Je tiens à remercier ici, pour nos nombreux échanges informels sur le sujet, mes correcteurs indéfectibles, Jean-Jacques Fleury, Maïté Goulliard et Sarah Reemers.

100. - PROST, Antoine, WINTER, Jay, op. cit., p. 287.

\section{ABSTRACTS}

By analysing the displays in eight museums in north-eastern Europe in 2013, prior to the centenary commemorations, different museum approaches can be compared. Some of these museums have been completely renovated for the event. The comparison is concerned by the way different museums offer displays of an aesthetic or immersive nature, but beyond these differences, the main issue is the measurement of how the permanent exhibitions are under the influence of national historiographies and contemporary national politics. Most of the themes and narratives presented are of a similar nature (the soldier at the front, the cultures of war, and so on) but others are approached from different viewpoints and dealt with in different emotional moods.

Par l'analyse des scénographies et des objets exposés dans huit musées de l'Europe du NordOuest, sont comparées différentes muséographies de la Grande Guerre en 2013, juste avant le centenaire. Une partie de ces musées viennent d'être complètement rénovés à l'occasion de cet événement international. Au-delà du choix des concepteurs de réaliser des muséographies esthétisantes ou immersives, l'enjeu principal est tout d'abord de mesurer l'influence potentielle des historiographies nationales et les différents contextes politiques sur les actuelles expositions permanentes. Enfin, la plupart des thèmes et des récits sont largement similaires (les soldats et le front ; les « cultures de guerres »), mais d'autres sont traités avec des points de vue et ambiances émotionnelles différenciés.

\section{INDEX}

Mots-clés: muséographie, musée, Première Guerre mondiale, Europe, Deutsches Historisches Museum (Berlin), Militärhistorisches Museum der Bundeswehr (Dresde), Imperial War Museum (Londres), musée royal de l'Armée (Bruxelles), In Flanders Fields Museum (Ypres), musée de la Grande Guerre du Pays de Meaux (Meaux), musée de l'Armée - Hôtel national des Invalides (Paris), Historial de la Grande Guerre (Péronne)

Keywords: Museography, museums, First World War, Europe, Deutsches Historisches Museum (Berlin), Milirärhistorisches Museum der Bundeswehr (Dresden), Imperial War Museum (London), musée royal de l'Armée (Brussels), In Flanders Fields Museum (Ypres), musée de la Grande Guerre du Pays de Meaux (Meaux), musée de l'Armée - Hôtel national des Invalides (Paris), Historial de la Grande Guerre (Péronne) 
AUTHOR

CÉLIA FLEURY

Département du Nord et IRHiS (CNRS - Lille 3), Responsable du développement des musées thématiques celia.fleury@cg59.fr 